\title{
Early-type stars in the young open cluster NGC 2244 and in the Monoceros OB2 association ${ }^{\star}$
}

\section{The multiplicity of O-type stars ${ }^{\star \star}$}

\author{
L. Mahy ${ }^{1}$, Y. Nazé ${ }^{1, \star \star \star}$, G. Rauw ${ }^{1, \dagger}$, E. Gosset ${ }^{1, \ddagger}$, M. De Becker ${ }^{1, \star \star}$, H. Sana ${ }^{2}$, and P. Eenens ${ }^{3}$ \\ 1 Astrophysical Institute, Liège University, Bât. B5C, Allée du 6 Août 17, 4000 Liège, Belgium \\ e-mail: mahy@astro.ulg.ac.be \\ 2 European Southern Observatory, Alonso de Cordova 1307, Casilla 19001, Santiago 19, Chile \\ 3 Departamento de Astronomia, Universidad de Guanajuato, Apartado 144, 36000 Guanajuato, GTO, Mexico \\ Received 14 January 2009 / Accepted 27 April 2009
}

\section{ABSTRACT}

\begin{abstract}
Aims. We present the results obtained from a long-term spectroscopic campaign to study the multiplicity of O-type stars in both the young open cluster NGC 2244 and the Mon OB2 association.

Methods. Our spectroscopic monitoring was performed over several years, allowing us to investigate different timescales. For each star, several spectral diagnostic tools were applied to search for line shifts and profile variations. We also measured the projected rotational velocity and revisited the spectral classification.

Results. Several stars in our sample have been previously considered to be spectroscopic binaries, although only a few scattered observations were available. Our results now have identified a more complex situation for two new spectroscopic binaries (HD 46149 in NGC 2244 and HD 46573 in Mon OB2). The first object is a long-period double-lined spectroscopic binary, although the exact value of its period remains uncertain and the second object is classified as an SB1 system with a period of about 10.67 days but the time series of our observations do not enable us to derive a unique orbital solution for this system. We also find another star to be variable in radial velocity (HD 46150 ) and detect line profile variations in two rapid rotators (HD 46056 and HD 46 485).

Conclusions. This spectroscopic investigation places a firm lower limit (17\%) on the binary fraction of O-stars in NGC 2244 and reveals the lack of short-period $\mathrm{O}+\mathrm{OB}$ systems in this cluster. In addition, a comparison of these new results with two other wellstudied clusters (NGC 6231 and IC 1805) puts forward possible hints of a relation between stellar density and binarity, which could provide constraints on the theories of the formation and early evolution of hot stars.
\end{abstract}

Key words. stars: binaries: spectroscopic - stars: early-type - galaxy: open clusters and association: individual: NGC 2244 galaxy: open clusters and association: individual: Monoceros OB2

\section{Introduction}

Since O-type stars are preferentially found in either young open clusters or OB associations, these two environments appear to play a key role in massive star formation. Detailed observations could provide interesting constraints on the different formation scenarios proposed by theoreticians (for a summary of these, see e.g., Zinnecker \& Yorke 2007). Massive stars in clusters are ideal targets because they represent a homogeneous population (of similar distance, age, and chemical composition). Moreover, the study of O-type stars in open clusters, and in particular their multiplicity, is of major interest for understanding the physical properties of these objects. Other parameters (e.g., orbital period, eccentricity, and mass ratio) of these multiple systems in addition to their binary frequency can provide a unique signature

* Table 2 is only available in electronic form at the CDS via anonymous ftp to cdsarc.u-strasbg.fr (130.79.128.5) or via http://cdsweb.u-strasbg. fr/cgi-bin/qcat?]/A+A/502/937

$\star \star$ Based on observations collected at Observatoire de Haute-Provence (France), San Pedro Màrtir Observatory (Mexico), La Silla Observatory (European Southern Observatory), and Asiago Observatory (Italy).

$\star \star \star$ Postdoctoral Researcher F.R.S.-FNRS.

Research Associate F.R.S.-FNRS.

* Senior Research Associate F.R.S.-FNRS. of star formation and dynamical interactions within the cluster during the very early phases of its existence.

Investigations based upon extensive spectroscopic campaigns in some clusters (e.g., NGC 6231, Sana et al. 2008; IC 1805, De Becker et al. 2006) have been performed over several years. These detailed observations led to a serious correction of the results proposed by García \& Mermilliod (2001) for O-type star rich clusters, by greatly improving our knowledge of the precise physical parameters of the binaries in these clusters. To extend these studies, we undertook a similar spectroscopic monitoring of the O-type star population in another young open cluster, NGC 2244.

With an inferred age of about 2-3 Myr (Chen et al. 2007) and a radial velocity (RV) of $26.2 \pm 3.4 \mathrm{~km} \mathrm{~s}^{-1}$ (Kharchenko et al. 2005), NGC 2244 is inside the Rosetta nebula and forms the core of the Monoceros OB2 association. The cluster is the youngest of two or three subgroups of OB stars and stellar aggregates in this association (Li \& Smith 2005).

Some stars in NGC 2244 were observed by Plaskett \& Pearce (1931) and reanalyzed by Petrie \& Pearce (1961). These authors found that the majority of these objects has quite constant RVs. These results were, however, subsequently challenged by Abt \& Biggs (1972) and Liu et al. (1989).

From a compilation of heterogeneous data, García \& Mermilliod (2001) estimated the proportion of spectroscopic 
binaries in NGC 2244 to be about $50 \%$ of the O-type stars (three out of six considered as binaries). Such an O-star binary fraction would make this cluster the third binary-rich cluster in their sample after NGC 6231 and IC 1805. As already mentioned above, the figures for these two clusters were subsequently significantly revised (Rauw \& De Becker 2004; De Becker et al. 2006; Sana et al. 2008). In addition, until now, only two spectroscopic binaries have been detected with certainty in Mon OB2: HD 47129 (Plaskett's star, Linder et al. 2008) and HD 48099 (Stickland 1996; Mahy et al. in preparation) which both lie outside NGC 2244. These conflicting results provide support for a revised, thorough analysis of the binarity in NGC 2244.

Other studies in different wavelength domains have provided additional information about the content and overall organization of the cluster. Chandra mosaic observations of NGC 2244 were presented by Wang et al. (2008). The X-ray spectra of the OB-type stars were found to be rather soft and consistent with the standard model of small-scale shocks produced, in the inner regions of the stellar winds, by the line-driving instability (Feldmeier et al. 1997). The X-ray luminosities of these stars follow the canonical $L_{\mathrm{X}} / L_{\mathrm{bol}}$ relation (Sana et al. 2006b) rather closely and found no evidence of extra emission caused by wind-wind collisions in binary systems. Wang et al. (2008) found that the fainter X-ray sources are strongly concentrated around HD $46150(\mathrm{O} 5.5 \mathrm{~V})$ whilst no strong clustering of $\mathrm{X}$-ray sources was found around the most massive component HD 46223 (O4 V). Following these authors, there are two possible explanations of this difference: either HD 46223 was ejected as a result of dynamical interactions (but it does not display a strong proper motion), or it may actually be younger and would then not be part of the same population as the central part of the cluster.

The present paper, based on a long-term spectroscopic campaign, is organized as follows. Section 2 describes the observing campaign. The properties of the individual objects (spectral classification, projected rotational velocity, and multiplicity) are presented in Sect. 3. The multiplicity and the rotational velocities of massive stars in NGC 2244 are discussed in Sect. 4 while Sect. 5 provides a summary of our results.

\section{Observations and data reduction}

Our team have collected 207 spectra of 9 stars over 9 years using several different telescopes. This sample has allowed us to investigate the presence of short and long-term variations for each star.

Most of our observations were obtained with the $1.52 \mathrm{~m}$ telescope of the Observatoire de Haute-Provence (OHP) equipped with the Aurélie spectrograph. The detector used was a thin back-illuminated CCD with $2048 \times 1024$ pixels of $13.5 \mu \mathrm{m}^{2}$. The grating \#3 (600 lines/mm) allowed us to obtain spectra with a resolving power of 10000 , centered on $5700 \AA$ and covering wavelengths between $5500 \AA$ and $5920 \AA$. Exposure times were typically 10 to $45 \mathrm{~min}$ resulting in signal-to-noise ratios higher than 150. Such high quality observations were needed to search for weak variations or the spectroscopic signature of faint companions. We also collected spectra with the same instrument in the blue domain (centered at $4670 \AA$ and covering the $4450-4900 \AA$ region). These data were acquired during 28 days in November 2007 and 6 days in September and October 2008. The entire dataset was reduced in a classical way (bias and flatfield corrections) using MIDAS software developed at ESO (European Southern Observatory). The spectra were wavelength calibrated using Thorium-Argon comparison spectra obtained immediately before or after the stellar spectrum. The spectra were finally normalized to the continuum by fitting polynomials of degree 4 or 5 .

Additional spectra were obtained at Observatorio Astronómico Nacional of San Pedro Màrtir (SPM), in Mexico, with the $2.1 \mathrm{~m}$ telescope equipped with Espresso. This échelle spectrograph gives 27 orders in the 3780-6950 $\AA$ wavelength domain with a resolving power of $R=18000$. The CCD detector was a SITE 3 optical chip with $1024 \times 1024$ pixels of $24 \mu \mathrm{m}^{2}$. Typical exposure times ranged from 5 to $15 \mathrm{~min}$. We had to add consecutive spectra of a given night at the expense of time resolution to obtain signal-to-noise ratios close to 300 . The data were reduced using the échelle package available within the MIDAS software.

In addition, we gathered spectra from both Elodie and ESO archives. Elodie was an échelle spectrograph giving 67 orders in the 3850-6850 $\AA$ spectral range $(R=42000)$ mounted at the $1.93 \mathrm{~m}$ telescope at OHP. We retrieved 13 spectra acquired between November 1999 and November 2005. The mean signalto-noise ratio of the data of our targets was 150 for an exposure time ranging from $10 \mathrm{~min}$ to $1 \mathrm{~h}$. The ESO archives provided us with 11 spectra taken with FEROS (Fiber-fed Extended Range Optical Spectrograph) mounted at the ESO/MPG $2.20 \mathrm{~m}$ telescope at La Silla $(R=48000)$. The data reduction was performed using a modified FEROS pipeline working under the MIDAS environment. Beyond the modifications already described in Sana et al. (2006a), several new features were implemented. We used a 2D fit of the order position for improved stability. We also used the wavelength calibration frames obtained with the "new" ThArNe calibration lamp (see e.g., FEROS-II user manual 77.0, Sect. 2.5). The latter however heavily saturates in the red, even on the shortest exposures planned by the calibration plan $^{1}$. Therefore, we computed a master calibration frame for each exposure time. We then proceeded with the extraction of the calibration spectra separately for each master frame. We finally used the detected lines from the $3 \mathrm{~s} / 15 \mathrm{~s} / 30 \mathrm{~s}$ master wavelength calibration frames to calibrate the orders 30-39/10-29/19 respectively. The saturated lines or the lines outside the linearity range of the detector were rejected from the fit and the detection threshold was optimised for each order. Finally, a 2iteration 3- $\sigma$ clipping method was used to discard the very few remaining discordant lines (mostly because of artifacts related to poorly corrected bad column effects). The obtained wavelength calibration residuals were all in the range 2.7-2.9 $\mathrm{m} \AA$.

To increase the dataset of HD 46149, we retrieved two spectra from Asiago archives of mean signal-to-noise ratio of about 155. These two spectra were taken by the $1.82 \mathrm{~m}$ Copernicus telescope equipped with the échelle instrument $(R=$ 28600 ). Unfortunately, no flatfields were available and we could only apply an overscan correction. Because of the lack of a wellsuited calibration atlas, the spectra were approximatively calibrated in wavelength but this first order calibration was subsequently checked using the interstellar lines. The overview of the observations is given in Table 1 (available electronically).

For most stars in NGC 2244, the radial velocities (RVs) and the equivalent widths $(E W s)$ were determined by fitting Gaussians to the line profiles, except for the two rapid rotators. Indeed, Gaussian functions were found to reproduce well the observed profile of moderately rotationally broadened lines in

\footnotetext{
1 As a reminder, the latter provides 3 series of two exposures with increasing exposure times of $3 \mathrm{~s}, 15 \mathrm{~s}$, and $30 \mathrm{~s}$, a scheme that is repeated at the beginning and at the end of the night.
} 
Table 1. Observations used for the study of massive stars in both NGC 2244 and Mon OB2.

\begin{tabular}{|c|c|c|c|c|c|c|}
\hline Object & Cluster & Obs. run & Telescope/Instrument & Wavelength domain & n\# Spectra & $\Delta T$ \\
\hline \multirow[t]{5}{*}{ HD 46056} & \multirow[t]{5}{*}{ NGC 2244} & Feb. 2006 & OHP - AURELIE/1.52 m & \multirow[t]{2}{*}{$5500-5900 \AA$} & 6 & 5.02 \\
\hline & & Apr. 2007 & $\mathrm{SPM}$ - ESPRESSO/2.20 m & & 5 & 6.00 \\
\hline & & Nov. 2007 & $\mathrm{OHP}$ - AURELIE/1.52 m & $4450-4900 \AA$ & 1 & \\
\hline & & Jan. 2008 & OHP - AURELIE/1.52 m & $5500-5900 \AA$ & 4 & 3.00 \\
\hline & & Mar. 2008 & SPM - ESPRESSO/2.20 m & & 1 & $\ldots$ \\
\hline \multirow[t]{13}{*}{ HD 46149} & \multirow[t]{13}{*}{ NGC 2244} & Dec. 1994 & Asiago - Echelle $/ 1.82 \mathrm{~m}$ & & 1 & $\ldots$ \\
\hline & & Dec. 1998 & Asiago - Echelle/1.82 m & & 1 & $\ldots$ \\
\hline & & Jan. 2003 & OHP - ELODIE/1.93 m & & 1 & $\ldots$ \\
\hline & & Nov. 2005 & OHP - ELODIE/1.93 m & & 1 & $\ldots$ \\
\hline & & Jan. 2006 & $\mathrm{ESO}-\mathrm{FEROS} / 2.20 \mathrm{~m}$ & & 1 & $\ldots$ \\
\hline & & Feb. 2006 & OHP - AURELIE/1.52 m & $5500-5900 \AA$ & 6 & 5.10 \\
\hline & & Apr. 2007 & SPM - ESPRESSO/2.20 m & & 3 & 3.97 \\
\hline & & Apr. 2007 & OHP - AURELIE/1.52 m & $5500-5900 \AA$ & 1 & $\ldots$ \\
\hline & & Nov. 2007 & OHP - AURELIE/1.52 m & $4450-4900 \AA$ & 6 & 25.05 \\
\hline & & Jan. 2008 & OHP - AURELIE/1.52 m & $5500-5900 \AA$ & 4 & 5.03 \\
\hline & & Mar. 2008 & SPM - ESPRESSO/2.20 m & & 5 & 4.93 \\
\hline & & Sep. 2008 & OHP - AURELIE/1.52 m & $4450-4900 \AA$ & 1 & $\ldots$ \\
\hline & & Oct. 2008 & OHP - AURELIE/1.52 m & $4450-4900 \AA$ & 3 & 4.02 \\
\hline \multirow[t]{11}{*}{ HD 46150} & \multirow[t]{11}{*}{ NGC 2244 } & Nov. 1999 & OHP - ELODIE/1.93 m & & 1 & $\ldots$ \\
\hline & & Jan. 2003 & OHP - ELODIE/1.93 m & & 1 & $\ldots$ \\
\hline & & Nov 2004 & OHP - ELODIE/1.93 m & & 1 & $\ldots$ \\
\hline & & Jan. 2006 & $\mathrm{ESO}-\mathrm{FEROS} / 2.20 \mathrm{~m}$ & & 1 & $\cdots$ \\
\hline & & Feb. 2006 & $\mathrm{ESO}-\mathrm{FEROS} / 2.20 \mathrm{~m}$ & & 1 & $\ldots$ \\
\hline & & Feb. 2006 & OHP - AURELIE/1.52 m & $5500-5900 \AA$ & 6 & 5.10 \\
\hline & & Apr. 2007 & SPM - ESPRESSO/2.20 m & & 5 & 5.99 \\
\hline & & Nov. 2007 & OHP - AURELIE/1.52 m & $4450-4900 \AA$ & 5 & 25.07 \\
\hline & & Jan. 2008 & OHP - AURELIE/1.52 m & $5500-5900 \AA$ & 4 & 2.99 \\
\hline & & Mar. 2008 & SPM - ESPRESSO/2.20 m & & 5 & 3.95 \\
\hline & & Oct. 2008 & OHP - AURELIE/1.52 m & $4450-4900 \AA$ & 4 & 4.00 \\
\hline \multirow[t]{5}{*}{ HD 46202} & \multirow[t]{5}{*}{ NGC 2244} & Jan. 2006 & ESO - FEROS/2.20 m & & 1 & $\ldots$ \\
\hline & & Feb. 2006 & OHP - AURELIE/1.52 m & $5500-5900 \AA$ & 2 & 1.98 \\
\hline & & Apr. 2007 & OHP - AURELIE/1.52 m & $5500-5900 \AA$ & 2 & 5.00 \\
\hline & & Nov. 2007 & OHP - AURELIE/1.52 m & $4450-4900 \AA$ & 4 & 20.00 \\
\hline & & Jan. 2008 & OHP - AURELIE/1.52 m & $5500-5900 \AA$ & 4 & 3.01 \\
\hline \multirow[t]{10}{*}{ HD 46223} & NGC 2244 & Jan. 1999 & OHP - ELODIE/1.93m & & 1 & $\ldots$ \\
\hline & & Nov 2005 & OHP - ELODIE/1.93 m & & 1 & $\ldots$ \\
\hline & & Jan. 2006 & ESO - FEROS $/ 2.20 \mathrm{~m}$ & & 1 & $\ldots$ \\
\hline & & Feb. 2006 & ESO - FEROS/2.2 0m & & 1 & $\ldots$ \\
\hline & & Feb. 2006 & OHP - AURELIE/1.52 m & $5500-5900 \AA$ & 6 & 5.03 \\
\hline & & Apr. 2007 & OHP - AURELIE/1.52 m & $5500-5900 \AA$ & 3 & 6.00 \\
\hline & & Nov. 2007 & OHP - AURELIE/1.52 m & $4450-4900 \AA$ & 5 & 21.98 \\
\hline & & Jan. 2008 & OHP - AURELIE/1.52 m & $5500-5900 \AA$ & 4 & 3.06 \\
\hline & & Mar. 2008 & SPM - ESPRESSO/2.20 m & & 3 & 2.01 \\
\hline & & Oct. 2008 & OHP - AURELIE/1.52 m & $4450-4900 \AA$ & 1 & $\ldots$ \\
\hline HD 46485 & NGC 2244 & Nov. 2005 & OHP - ELODIE/1.93 m & & 1 & $\begin{array}{ll}\ldots \\
\cdots\end{array}$ \\
\hline & & Jan. 2006 & ESO - FEROS $/ 2.20 \mathrm{~m}$ & & 1 & $\cdots$ \\
\hline & & Nov. 2007 & OHP - AURELIE/1.52 m & $4450-4900 \AA$ & 6 & 14.99 \\
\hline & & Jan. 2008 & OHP - AURELIE/1.52 m & $5500-5900 \AA$ & 4 & 3.05 \\
\hline & & Mar. 2008 & SPM - ESPRESSO/2.20 m & & 5 & 4.02 \\
\hline HD 46573 & Mon OB2 & Jan. 2006 & $\mathrm{ESO}-\mathrm{FEROS} / 2.20 \mathrm{~m}$ & & 1 & $\bar{\cdots}$ \\
\hline & & Feb. 2006 & ESO - FEROS/2.20 m & & 1 & $\ldots$ \\
\hline & & Feb. 2006 & OHP - AURELIE/1.52 m & $5500-5900 \AA$ & 3 & 2.97 \\
\hline & & Apr. 2007 & SPM - ESPRESSO/2.20 m & & 5 & 5.99 \\
\hline & & Nov. 2007 & OHP - AURELIE/1.52 m & $4450-4900 \AA$ & 6 & 25.98 \\
\hline & & Jan. 2008 & OHP - AURELIE/1.52 m & $5500-5900 \AA$ & 5 & 3.05 \\
\hline & & Oct. 2008 & OHP - AURELIE/1.52 m & $4450-4900 \AA$ & 3 & 3.00 \\
\hline HD 46966 & Mon OB2 & Jan. 2003 & OHP - ELODIE/1.93m & & 2 & 1.76 \\
\hline & & Nov 2004 & OHP - ELODIE/1.93 m & & 1 & $\ldots$ \\
\hline & & Jan. 2006 & ESO - FEROS/2.20 m & & 2 & 1.94 \\
\hline & & Feb. 2006 & OHP - AURELIE/1.52 m & $5500-5900 \AA$ & 6 & 5.01 \\
\hline & & Feb. 2007 & OHP - AURELIE/1.52 m & $5500-5900 \AA$ & 1 & $\ldots$ \\
\hline & & Apr. 2007 & OHP - AURELIE/1.52 m & $5500-5900 \AA$ & 1 & $\ldots$ \\
\hline & & Nov. 2007 & OHP - AURELIE/1.52 m & $4450-4900 \AA$ & 5 & 24.05 \\
\hline & & Jan. 2008 & OHP - AURELIE/1.52 m & $5500-5900 \AA$ & 4 & 3.02 \\
\hline HD 48279 & Mon OB2 & Jan. 2000 & OHP - ELODIE/1.93m & & 1 & $\ldots$ \\
\hline & & Nov 2004 & OHP - ELODIE/1.93 m & & 1 & $\ldots$ \\
\hline & & Nov. 2007 & OHP - AURELIE/1.52 m & $4450-4900 \AA$ & 9 & 16.02 \\
\hline & & Jan. 2008 & OHP - AURELIE/1.52 m & $5500-5900 \AA$ & 4 & 3.01 \\
\hline & & Mar. 2008 & SPM - ESPRESSO/2.20 m & & 4 & 4.01 \\
\hline & & Oct. 2008 & OHP - AURELIE/1.52 m & $4450-4900 \AA$ & 1 & $\ldots$ \\
\hline
\end{tabular}

The first and the second columns give the name of the object and the membership to the cluster or association (Ogura \& Ishida 1981). The next columns are the epochs of the campaign, the telescope/instrument used, the observed wavelength domain, the number of spectra obtained and the time elapsed between the first and the last spectrum of the run, expressed in days. 
massive stars. To measure the RVs, we fitted the bottom half of the line profiles, while the entire line was used to estimate the $E W$ s. For the two rapid rotators, we used a synthetic line profile generated with the projected rotational velocity of the star to determine the Doppler shifts, and we integrated the complete line profiles to measure the $E W \mathrm{~s}$. We note that we used the same rest wavelengths as Conti et al. (1977) to calculate the RVs.

We inferred the spectral type and the luminosity class of each star by using the classification criteria of Conti \& Alschuler (1971), Conti (1973), and Mathys (1988, 1989) hereafter referred to as "Conti's criteria". To support our results, we visually compared the spectra to the atlas of Walborn \& Fitzpatrick (1990). We also checked the luminosity class by comparing the computed visual absolute magnitude with the photometric calibration proposed by Martins \& Plez (2006). We also needed to assume a value for the distance of the cluster. Massey et al. (1995) derived a spectroscopic parallax to this region of $1.9 \pm 0.1 \mathrm{kpc}$, whereas more recent photometric studies place NGC 2244 at a distance of between 1.4 and $1.7 \mathrm{kpc}$ (Hensberge et al. 2000) in agreement with previous estimates (Ogura \& Ishida 1981; Pérez 1991). In the present paper, we adopt a mean distance for NGC 2244 of $1.55 \mathrm{kpc}$. However, we note that a change of distance by $10 \%$ would not modify our conclusions. Finally, we compared our spectral classification with previous results from earlier studies (notably quoted in the Reed catalogue Reed 2005).

\section{The O-type star population in NGC 2244 and Mon OB2}

Our study of the multiplicity of O-type stars is based on two wavelength domains: a blue region (4450-4900 A) and a yellow one (5500-5920 $\AA$ ). In addition to the RV and profile analysis, the former setting provides an accurate determination of the spectral classification of each star; the numerous interstellar features observed in the latter setting enable us to check the quality of the wavelength calibration and RV measurements. We provide, in Table 2 (available electronically at CDS), the heliocentric Julian days (HJD) and the RVs of each spectrum, while Table 3 quotes, object by object, the mean RVs and the 1- $\sigma$ standard deviation of the different lines.

In the following, an object is considered to be a true spectroscopic binary (Sect.3.1) if we detect either the presence of significant, periodic RV variations or the signature of a companion whose spectral lines move in anti-phase with those of the main star. If a star fulfills the former criterion, a full orbital solution can be computed, leading to the precise determination of the orbital parameters. When the latter criterion is fulfilled, it only allows us to evaluate the spectral type of the two components and their mass ratio, with only loose constraints on the orbital period. If none of these criteria is fulfilled, but significant RV variations are detected, then the star will be considered to be a "RV variable", and thus a binary candidate. The RV variations are considered as significant if they are larger or equal to three times the average RV measurement error. For all stars except the two rapid rotators, this error is well represented (as was confirmed by tests on individual data) by the RV dispersion in the narrow diffuse interstellar band (DIB) at $\lambda$ 5780, which has similar strength and width to the stellar lines. For the rapid rotators, $\chi^{2}$ evaluation from shifts of the synthetic line profile showed the $\mathrm{RV}$ error to be about $10 \mathrm{~km} \mathrm{~s}^{-1}$. An object for which significant $\mathrm{RV}$ variations are detected is presented in Sect. 3.2. The remaining objects are presented in Sect. 3.3.
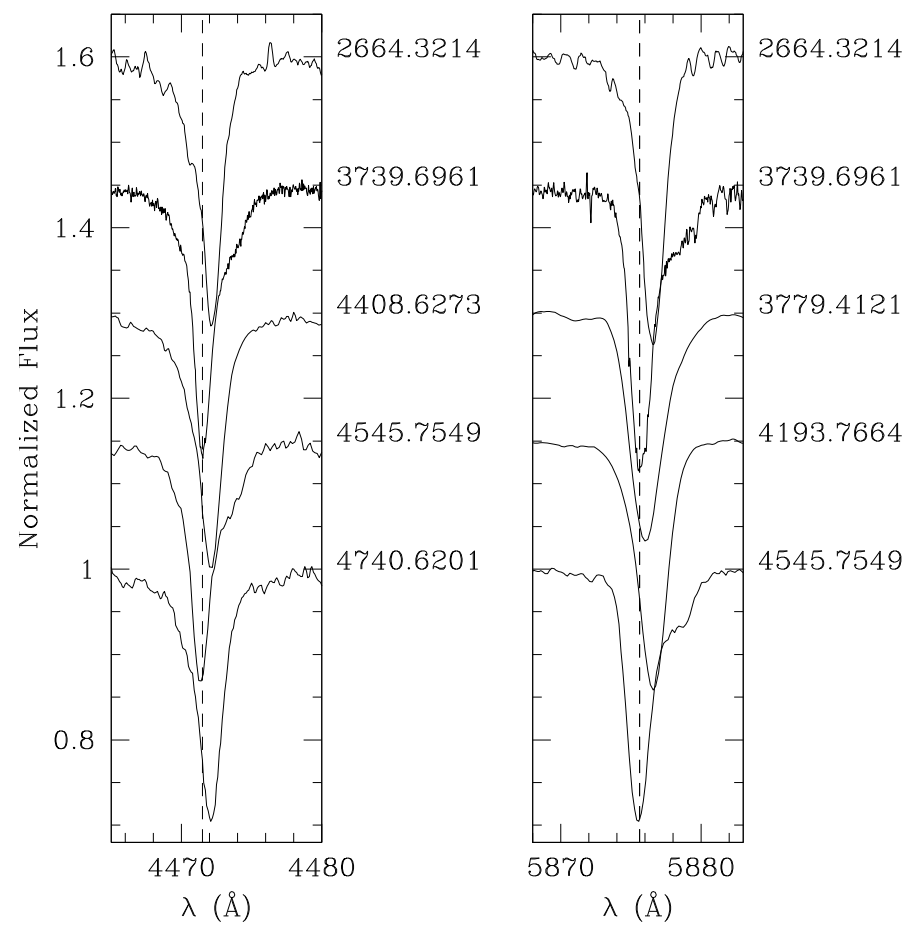

Fig. 1. He I $\lambda 4471$ and He I $\lambda 5876$ line profiles of HD 46149 observed during our spectroscopic campaigns. The time is given on the right side of the figure in the format HJD - 2450000 . All spectra are displayed in the heliocentric frame of reference. The vertical dashed line represents the rest wavelength. The signature of the secondary is clearly seen, in particular at HJD 2454545.

\subsection{Spectroscopic binaries}

\subsubsection{HD 46149}

The first spectroscopic study of HD 46149 was completed by Plaskett \& Pearce (1931), who measured a mean RV of about $45.0 \pm 1.5 \mathrm{~km} \mathrm{~s}^{-1}$. On the basis of new data, Petrie \& Pearce (1961) confirmed that the star might be single. They obtained a mean RV of about $38.0 \pm 2.1 \mathrm{~km} \mathrm{~s}^{-1}$. Liu et al. (1989) analyzed three datasets obtained over three different epochs (in November 1987, February 1988, and October 1988). They found a RV dispersion of about $10.2 \mathrm{~km} \mathrm{~s}^{-1}$ and, therefore, considered the star to be a potential binary. Turner et al. (2008) reported some velocity variabilities of HD 46149 because of the presence of a close spectroscopic companion.

In February 2006, the measurements of RVs obtained for six spectra acquired over six consecutive nights did not find evidence of any significant variation $\left(\overline{R V}=21.6 \pm 2.1 \mathrm{~km} \mathrm{~s}^{-1}\right)$. Moreover, no variation was detected in the line profiles. However, if we compare these RVs with the April 2007 data (HJD 2454 199, in Fig. 1), we obtain a shift close to $25 \mathrm{~km} \mathrm{~s}^{-1}$ for the HeI $\lambda 5876$ line, and a RV shift of about $40 \mathrm{~km} \mathrm{~s}^{-1}$ for He I $\lambda 4471$ is measured between the data taken in January 2006 and in November 2007. Our analysis of the entire spectroscopic campaign for HD 46149 clearly shows significant RV shifts between the observing runs. The two extreme RV values in our sampling are approximately -5 (Jan. 2006, Mar. 2008) and $+50 \mathrm{~km} \mathrm{~s}^{-1}$ (Apr. 2007, Nov. 2007 and Sept.Oct. 2008). In addition, the FEROS spectrum and the data collected between January and April 2008 allowed us to detect the presence of a companion in the red wing of most of the He I lines (visible in Fig. 1 at, for example, HJD 2453739 and 
Table 3. The mean radial velocities ${ }^{a}$ and the $1-\sigma$ dispersions.

\begin{tabular}{|c|c|c|c|c|c|c|c|c|c|}
\hline Star & $\operatorname{He} \mathrm{I} \lambda 4471$ & He II $\lambda 4542$ & He II $\lambda 4686$ & O III $\lambda 5592$ & DIB $\lambda 5780$ & $\mathrm{C}$ IV $\lambda 5801$ & $\mathrm{C}$ IV $\lambda 5812$ & $\mathrm{He}$ I $\lambda 5876$ & $\mathrm{Na} \mathrm{I} \lambda 5890$ \\
\hline HD 46056 & $36.1 \pm 6.9$ & $49.6 \pm 9.3$ & $30.4 \pm 12.1$ & & $26.0 \pm 4.1$ & & & $28.7 \pm 9.7$ & $23.3 \pm 2.0$ \\
\hline HD 46149 & $27.3 \pm 21.8$ & $27.6 \pm 18.7$ & $28.4 \pm 22.0$ & $12.6 \pm 21.4$ & $23.5 \pm 4.7$ & $24.1 \pm 21.3$ & $20.7 \pm 20.3$ & $22.3 \pm 19.4$ & $21.4 \pm 3.0$ \\
\hline HD 46150 & $31.2 \pm 4.0$ & $45.3 \pm 8.1$ & $44.8 \pm 5.0$ & $26.9 \pm 7.8$ & $23.8 \pm 3.9$ & $48.8 \pm 11.7$ & $34.0 \pm 12.9$ & $32.6 \pm 5.4$ & $20.7 \pm 2.4$ \\
\hline HD 46202 & $39.8 \pm 3.1$ & $38.8 \pm 3.8$ & $38.0 \pm 3.2$ & $31.8 \pm 1.3$ & $25.4 \pm 1.5$ & $40.4 \pm 2.2$ & $36.1 \pm 2.2$ & $40.0 \pm 1.4$ & $23.5 \pm 1.2$ \\
\hline HD 46223 & $34.5 \pm 6.7$ & $44.5 \pm 3.2$ & $47.0 \pm 3.3$ & $31.1 \pm 7.4$ & $23.8 \pm 4.6$ & $50.9 \pm 2.9$ & $38.6 \pm 6.4$ & $35.6 \pm 2.7$ & $23.4 \pm 3.4$ \\
\hline HD 46485 & $31.5 \pm 7.5$ & $43.5 \pm 10.7$ & $34.0 \pm 9.4$ & & $21.8 \pm 5.5$ & & & $30.8 \pm 11.1$ & $21.3 \pm 4.0$ \\
\hline HD 46573 & $47.0 \pm 6.6$ & $48.4 \pm 10.0$ & $50.2 \pm 7.6$ & $47.9 \pm 8.5$ & $27.6 \pm 3.9$ & $59.5 \pm 5.7$ & $53.2 \pm 6.1$ & $50.3 \pm 5.4$ & $26.9 \pm 1.4$ \\
\hline HD 46966 & $39.2 \pm 1.4$ & $44.9 \pm 2.0$ & $45.3 \pm 2.0$ & $38.4 \pm 2.6$ & $23.4 \pm 4.7$ & $47.7 \pm 3.0$ & $43.4 \pm 3.3$ & $40.8 \pm 2.4$ & $20.8 \pm 2.2$ \\
\hline HD 48279 & $31.4 \pm 3.6$ & $40.6 \pm 2.6$ & $38.9 \pm 5.1$ & $32.8 \pm 11.8$ & $23.1 \pm 5.0$ & $43.3 \pm 6.0$ & $31.9 \pm 7.4$ & $39.5 \pm 2.1$ & $20.8 \pm 3.3$ \\
\hline
\end{tabular}

${ }^{a}$ The data are expressed in $\mathrm{km} \mathrm{s}^{-1}$.

HJD 2454545). The determination of the individual RVs enables us, in this case, to estimate the mass ratio of the two components. At the largest observed separation (at HJD 2454545 ), the highest precision on the RVs of both stars was obtained from analyzing the He I $\lambda 5876$ line, which yields velocities of -5.8 and $+143.3 \mathrm{~km} \mathrm{~s}^{-1}$ for the primary and the secondary, respectively. The RV of the blended spectrum, $+52.5 \mathrm{~km} \mathrm{~s}^{-1}$ at HJD $\sim 2452664$, was measured from the He I $\lambda 5876$ line. From these shifts, we estimate a mass ratio $\left(M_{1} / M_{2}\right)$ of about 1.6. We nevertheless emphasize that the phases for which we measured the RVs might not be the extreme ones of the orbital cycle. In consequence, our mass ratio computation must be considered preliminary at best. We also estimated the flux ratio between the primary and the secondary. We measured, from the deblended He I $\lambda 5876$ line, an $E W$ for the primary of $572 \mathrm{~m} \AA$, which we compared with the average $E W$ quoted by Conti (1973) for such stars. We obtained an optical flux ratio $L_{1} /\left(L_{1}+L_{2}\right)$ for the primary of about 0.7 . In consequence, the flux ratio $L_{1} / L_{2}$ is close to 2.3 . The obtained mass and flux ratios thus imply that the secondary is an early B-type (for comparison, from the stellar parameters listed in Martins et al. 2005, a binary system composed of an O8 V and an $09.5 \mathrm{~V}$ would have a theoretical mass ratio of $M_{1} / M_{2}=1.3$ and a flux ratio $L_{1} / L_{2}$ of about 1.9 ).

We then applied the Fourier analysis to the time series of the primary RVs of He I $\lambda 4471$, He II $\lambda 4542$, C IV $\lambda 5812$, and He I $\lambda 5876$ following the method described by Heck et al. (1985) and revised by Gosset et al. (2001). In all cases, we observed the highest peaks in the periodograms but their positiond varied according to the studied line. For example, the computed periodogram corresponding to $\mathrm{He} \mathrm{I} \lambda 5876$ yields a period of about 47 days while the one corresponding to C IV $\lambda 5812$ indicates a 1600 day period. We also performed a period search on the combined RV list of He I $\lambda 4471$ and He I $\lambda$ 5876. Even in this case, the detected peak in the periodogram is not significant enough to infer reliably a particular orbital period. The data sampling is thus too poor at the present time to constrain the period of HD 46149.

To separate the two components of HD46149, we used our disentangling program, based on the method proposed by González \& Levato (2006). This consists of an iterative process that uses alternately the spectrum of one component (shifted according to its radial velocity) to remove it from the observed spectra and then to calculate a mean spectrum of the other component. Furthermore, this technique allows us to compute the RVs of each star by cross-correlation even at phases for which the lines are heavily blended. We applied the disentangling algorithm to the wavelength domains 4000-4220 $\AA$ and 4450 $4730 \AA$. To create the RV cross-correlation mask, we used the
He I $\lambda$ 4026, Si IV $\lambda$ 4089, He I $\lambda$ 4120, He I $\lambda 4143$, He I $\lambda 4471$, $\operatorname{Mg}$ II $\lambda 4481$, and He I $\lambda 4713$ lines for the secondary, and we added the Si IV $\lambda 4116$, He II $\lambda$ 4200, and He II $\lambda 4542$ lines for the primary. The calculated mean spectrum of the secondary star looks similar to that of a B0 V star in the Walborn \& Fitzpatrick (1990) atlas. However, because of the poor sampling of the data, this result must be considered very preliminary.

HD 46149 was classified as an O8.5 V ((f)) star (Massey et al. 1995), but we revise its classification to O8 V. We note that this does not depend on the degree of blending in the spectra. With the mass ratio computed above and a theoretical mass for the primary star close to $22 M_{\odot}$ (Martins et al. 2005), we estimate the secondary mass to be approximately $14 M_{\odot}$. Therefore, the system probably consists of O8 V + B0-1 V stars: HD 46149 is the first $\mathrm{O}+\mathrm{B}$ binary discovered so far in NGC 2244.

\subsubsection{HD 46573}

This star is a member of Mon OB2, but does not belong to NGC 2244 (Turner 1976). According to the RV variability criterion, the RV dispersions for the stellar lines of HD 46573 are at the limit of being considered as significant. However, we computed the Temporal Variance Spectrum (TVS, Fullerton et al. 1996) from the Aurélie spectra for the He I $\lambda 4471$, He II $\lambda 4542$, C IV $\lambda \lambda$ 5801-12, and He I $\lambda 5876$ lines. In each case, we obtained double-peaked profiles (Fig. 2). Together, the RV dispersion and the double-peaked TVS structures lend significant support to the binary nature of the star, first envisaged by Mason et al. (1998).

We performed a Fourier analysis of the RVs. We independently applied this method to three RV time series (He I $\lambda 4471$, He II $\lambda$ 4542, and He I $\lambda$ 5876) but the semi-amplitude spectra did not allow us to identify any dominant peak likely to be associated with a well-determined period. We then combined the RVs of the He I $\lambda 4471$ and He I $\lambda 5876$ lines. The highest peak in the resulting semi-amplitude spectrum occurs at a frequency of $0.0937 \mathrm{~d}^{-1}$ (Fig. 3), which corresponds to a period of about 10.67 days but this value is still very preliminary since random fluctuations cannot be totally excluded. The SB1 orbital solution of HD 46573 was calculated with the program LOSP (Liège Orbital Solution Package, Sana \& Gosset, A\&A submitted) based on the method of Wolfe et al. (1967). We assigned the same weight, i.e., 1.0, to all spectra and fixed the period value to 10.67 days. Table 4 gives the main orbital elements computed for HD 46573. Folded with this period, our data favored a non-zero eccentricity (Fig. 4, bottom panel). While this is an insufficient number to calculate a full orbital solution, the RVs from He II and C IV lines support the results from the He I lines (Fig. 4). However, there are still some phase intervals without 

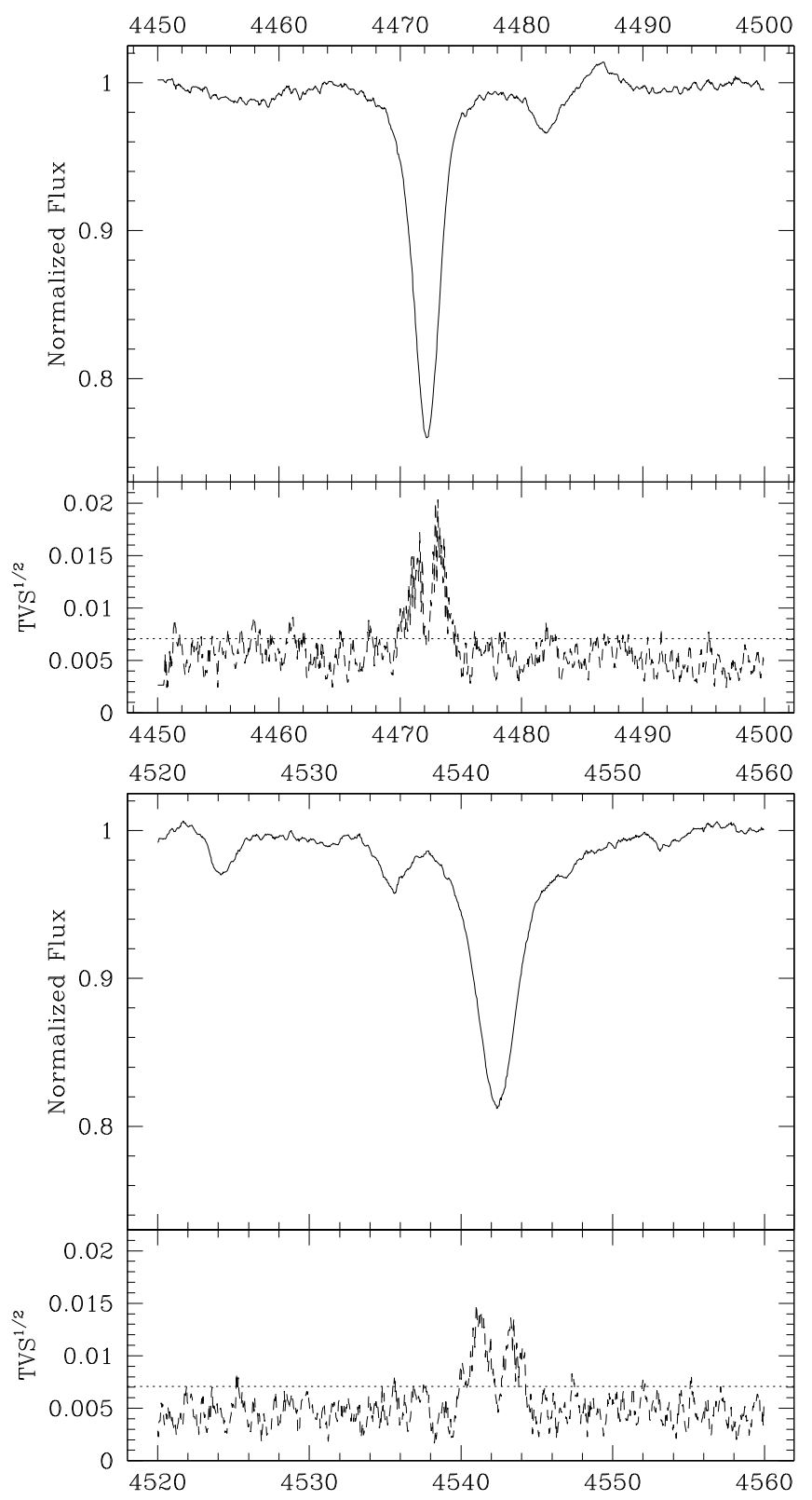

Fig. 2. The mean spectrum and TVS of HD 46573 computed from the Aurélie spectra for the He I $\lambda 4471$ (top) and He II $\lambda 4542$ lines (bottom). In both cases, the double peaked structure is clearly visible. The dotted line illustrates the $99 \%$ significance level for the variability evaluated following the approach of Fullerton et al. (1996).

observations and we therefore need more data to secure the period and the orbital parameters.

We then computed, with the Fourier transform method (Simón-Díaz \& Herrero 2007), the projected rotational velocity of HD 46573. Previously estimated to be about $95 \mathrm{~km} \mathrm{~s}^{-1}$ (Conti \& Ebbets 1977), we measured, for four lines (He II $\lambda 4686$, O III $\lambda$ 5592, C IV $\lambda$ 5801, and He I $\lambda$ 5876), a mean value of about $v \sin i=110 \pm 18 \mathrm{~km} \mathrm{~s}^{-1}$.

The spectral type was previously quoted as $07.5 \mathrm{~V}((\mathrm{f}))$ (Conti \& Leep 1974), O7 V (Bisiacchi et al. 1982), or O7 III ((f)) (Walborn 1971). In our data, the He I $\lambda 4471$ to He II $\lambda 4542$ ratio implies an O7.5 type, while the $E W$ ratio between Si IV $\lambda 4088$ and $\mathrm{He}$ I $\lambda 4143$ favors a main-sequence (MS) luminosity class. The visual absolute magnitude is more uncertain since the

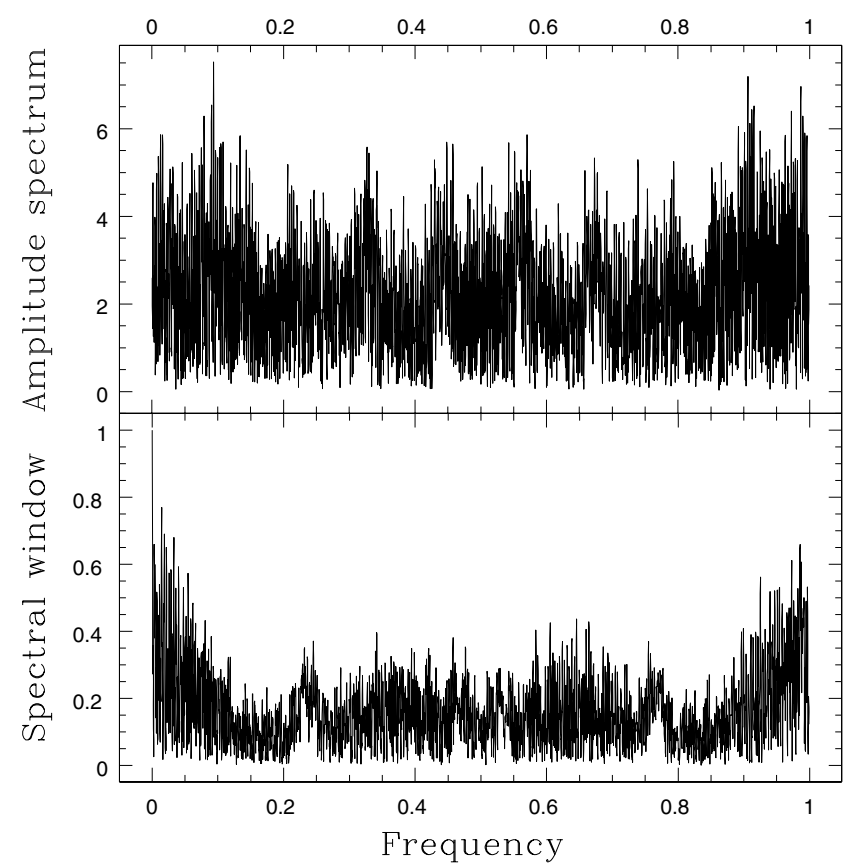

Fig. 3. The square root of the power spectrum (top) and the spectral window (bottom) of HD 46573 computed from RVs of both He I $\lambda 4471$ and $\lambda 5876$ lines. The $y$-axis corresponds to the semi-amplitude of the signal. The frequency is expressed in $\mathrm{d}^{-1}$.

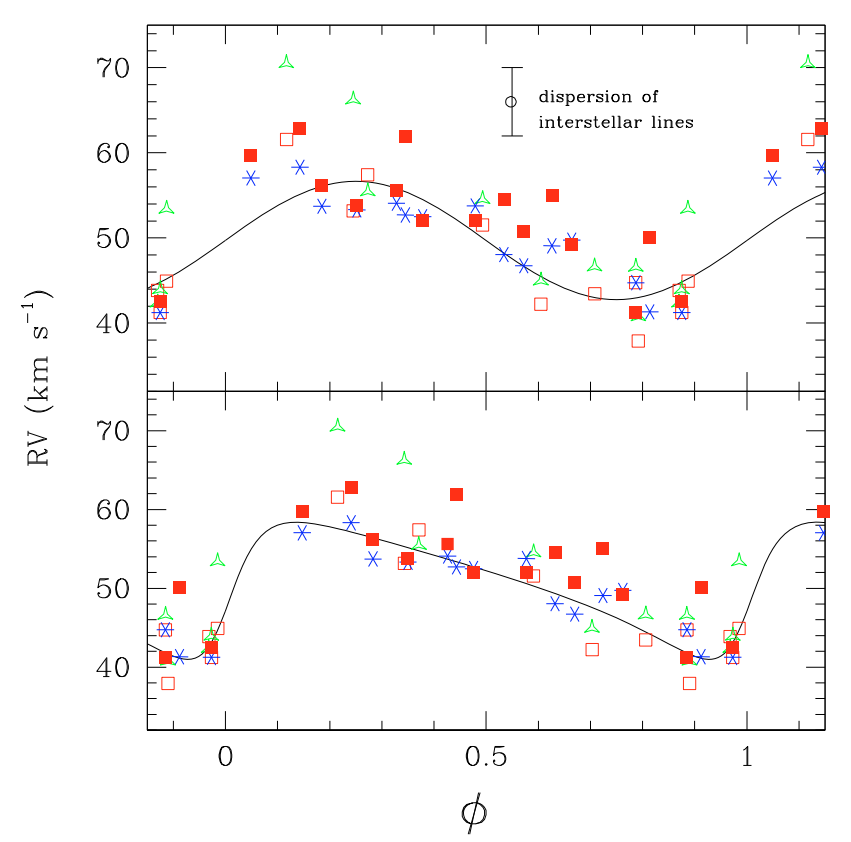

Fig. 4. Top: the best-fit circular radial velocity curve for HD 46573 computed from the RVs of the He I $\lambda 4471$ (open squares) and $\lambda 5876$ (filled squares) lines for an orbital period of 10.67 days. The open triangles correspond to the RVs of the He II $\lambda 4542$ line and the stars to the RVs of the CIV $\lambda 5812$ line. The error bar corresponds to the $\pm 1-$ $\sigma$ RV dispersion of the DIB $\lambda$ 5780. Bottom: the best-fit eccentric orbital solution for HD 46573 computed for the same two lines.

distance to the star is not exactly known. Assuming that Mon OB2 lies at the same distance as NGC 2244, we estimated $M_{V}$ for the two extreme values of the distance of NGC $2244(1.4 \mathrm{kpc}$ and $1.7 \mathrm{kpc})$. We obtain $M_{V}=-4.67$ and $M_{V}=-5.07$, using $V=7.933$ and $E(B-V)=0.61$ 
Table 4. Possible orbital parameters of the SB1 solution of HD $46573^{a}$.

\begin{tabular}{lcc}
\hline \hline Parameter & Circular & Eccentric \\
\hline$P$ (days) & 10.67 (fixed) & 10.67 (fixed) \\
$e$ & $\ldots$ & $0.47 \pm 0.13$ \\
$\omega$ (degrees) & $\ldots$ & $254.63 \pm 14.2$ \\
$T_{0}$ & $3732.24 \pm 0.26$ & $3731.68 \pm 0.48$ \\
$\gamma\left(\mathrm{km} \mathrm{s}^{-1}\right)$ & $49.7 \pm 0.7$ & $50.9 \pm 0.8$ \\
$K\left(\mathrm{~km} \mathrm{~s}^{-1}\right)$ & $6.9 \pm 0.9$ & $8.5 \pm 1.1$ \\
$a \sin i\left(R_{\odot}\right)$ & $1.46 \pm 0.19$ & $1.58 \pm 0.24$ \\
$f(m)\left(M_{\odot}\right)$ & $0.0003 \pm 0.0001$ & $0.0005 \pm 0.0002$ \\
rms $\left(\mathrm{km} \mathrm{s}^{-1}\right)$ & 3.5 & 2.6 \\
Prob $\left(\chi^{2}>\right)$ & $78 \%$ & $99 \%$ \\
\hline
\end{tabular}

${ }^{a} T_{0}$ (expressed in HJD-2450 000) refers to the time of periastron passage for $e \neq 0$ and to the conjunction with the primary star in front for $e=0 . \gamma, K$, and $a \sin i$ are, respectively, the systemic velocity, the semiamplitude of the radial velocity curve, and the projected separation between the center of the star and the center of mass of the binary system. The orbital elements are given for a circular and an eccentric orbit, respectively, by the RVs of both He I $\lambda 4471$ and $\lambda 5876$.

(Maíz-Apellániz et al. 2004). The reported results are closer to a MS star than to a giant (Martins \& Plez 2006). The spectrum also exhibits weak N III $\lambda \lambda$ 4634-41 emission lines and strong He II $\lambda 4686$ absorption. In $\mathrm{H} \alpha$, we detect weak emission in the absorption line probably related to the surrounding nebula. Thus, we favor an $07.5 \mathrm{~V}((\mathrm{f}))$ spectral type, for HD 46573.

In summary, two possible explanations exist for why the secondary component is not detected: a low-mass companion or a low inclination of the orbital plan. However, with such a large $v \sin i$ for the primary, it seems unlikely that the system is observed at such a low inclination angle. In consequence, we assume that HD 46573 is an O7.5 V ((f)) star, which possesses a lower mass companion.

\subsection{Radial velocity variables}

\subsubsection{HD 46150}

Reported as the second hottest star in NGC 2244, HD 46150 is also one of the most well studied objects in this cluster. This star was first observed by Plaskett \& Pearce (1931), who reported constant RVs. Since the 1970's, many other investigations have been devoted to HD 46150 (Abt \& Biggs 1972; Conti \& Ebbets 1977; Garmany et al. 1980), but the interpretations of the multiplicity of the star varied greatly from one author to another. Garmany et al. (1980) considered the changes in the RVs to be a consequence of motions in the stellar atmosphere. Liu et al. (1989) and Underhill \& Gilroy (1990) suggested that the star could be a spectroscopic binary, even though the signature of the secondary component had not yet been detected. Fullerton (1990) observed asymmetric line profiles, typical of an SB2.

Between November 1999 and October 2008, we collected 34 spectra spread over different timescales. We observed a slight RV variability in the absorptions (Fig. 5 notably compares the He II $\lambda 4542$ line at HJD 2453772 and HJD 2 454 397) as well as in the emission lines which is supported by the larger dispersion for stellar lines compared to the interstellar ones. For instance, we noted a RV shift of He II $\lambda 4542$ in the blue data by $28 \mathrm{~km} \mathrm{~s}^{-1}$ and of He I $\lambda 5876$ in the yellow spectra by $21 \mathrm{~km} \mathrm{~s}^{-1}$. Moreover, the RV dispersion is smaller for the He I lines than for the He II and metallic lines, which are clearly RV variable. The presence of an unresolved companion could explain this effect. If the secondary is cooler, the He I lines of the primary will
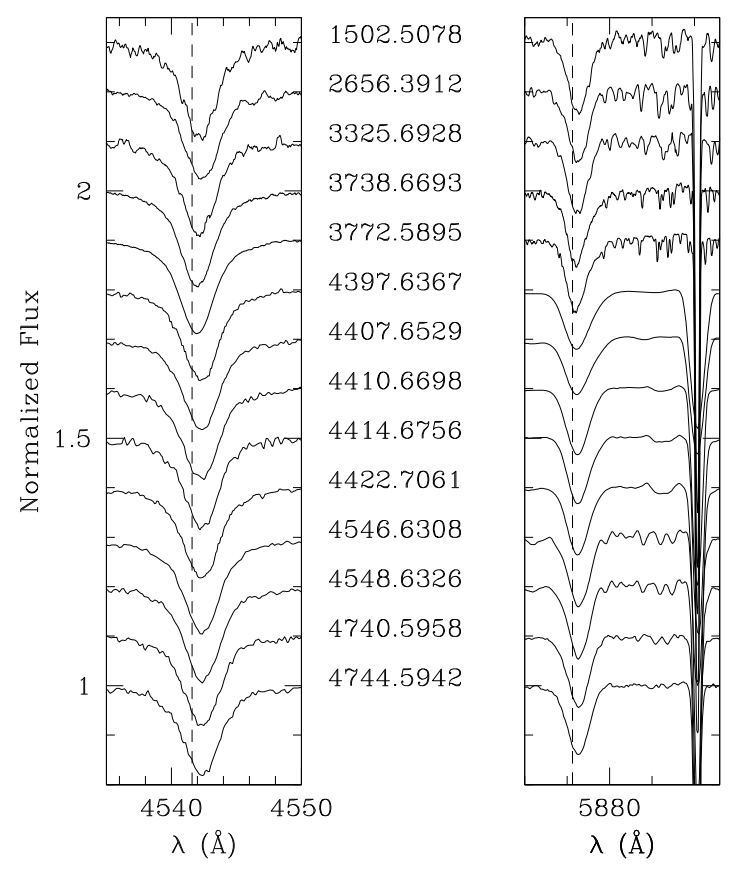

1502.5078 2656.3912 3325.6928 3738.6693 3772.5895 3777.3699 $3780.485 r$ 4193.6824 4196.6761 4199.6737 4472.3393 4475.3338 4546.6308 4548.6326

Fig. 5. He II $\lambda 4542$ and He I $\lambda 5876$ line profiles of HD 46150 observed during our spectroscopic campaigns.

indeed be more affected by blends with the lines of the secondary component. The RV variations in lines associated with a stronger ionization (He II, C IV ,...) thus reflect the true motion of the primary more accurately, leading to a larger RV dispersion.

To compute the TVS from all spectra of HD 46150, we had to degrade the spectral resolution of the échelle spectra to that of the Aurélie data. We proceeded by convolving the échelle spectra with Gaussians to ensure a homogeneous spectral resolution for all spectra. Variations in double peaks (in the He I $\lambda$ 4471, He II $\lambda 4542$, He II $\lambda$ 4686, and C IV $\lambda \lambda$ 580112 lines) and even variations in triple peaks, shown in Fig. 6, for the He I $\lambda 5876$ line, were observed. These features are reminiscent of what is usually found in spectroscopic binaries. However, no obvious signature of a companion could be found. We also note that these variations in double peaks are not visible on short timescales ( $\sim 7$ days), which implies that the system is a rather long-period binary.

To measure the period, we applied the Fourier method to the measured RVs. We used this technique independently for the He I $\lambda$ 4471, He II $\lambda$ 4542, C IV $\lambda$ 5812, and He I $\lambda 5876$ lines. For each line, several peaks are present in the periodogram but their semi-amplitude nearly corresponds to the standard deviation in the RVs of the interstellar lines. We also computed the semiamplitude spectrum by combining the RVs of the He I $\lambda 4471$ and the He I $\lambda 5876$ lines but, once again, the peak was insignificant to ascertain the period.

Previously estimated to be $118 \pm 25 \mathrm{~km} \mathrm{~s}^{-1}$ (Conti \& Ebbets 1977 ) and $86 \mathrm{~km} \mathrm{~s}^{-1}$ (Penny 1996), the projected rotational velocity of HD 46150 has been determined using the Fourier method for four different lines (He II $\lambda 4686$, O III $\lambda 5592$, $\mathrm{C} \operatorname{IV} \lambda$ 5801, and He I $\lambda$ 5876). We find a mean value of $v \sin i=$ $97 \pm 9 \mathrm{~km} \mathrm{~s}^{-1}$.

In the literature, the spectral type is quoted to be between O5 V ((f)) (Underhill \& Gilroy 1990; Maíz-Apellániz et al. 2004) and O5.5 V ((f)) (Conti \& Ebbets 1977; Garmany et al. 1980). From our measurements of the $E W \mathrm{~s}$, we confirm the 


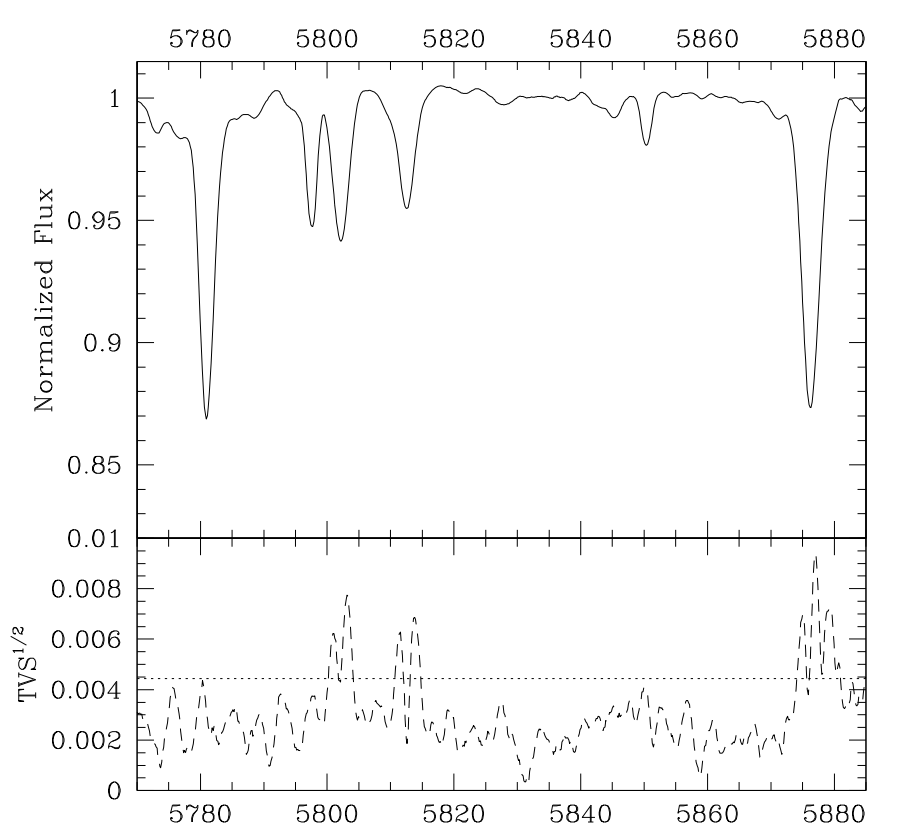

Fig. 6. Mean spectrum and TVS of HD 46150 for the C IV $\lambda \lambda$ 5801-12 and $\mathrm{He}$ I $\lambda 5876$ lines. The dotted line illustrates the $99 \%$ significance level of the variability evaluated following the approach of Fullerton et al. (1996). We can clearly see the double peaked structure in the stellar lines.

latter spectral type. We observe weak N III $\lambda \lambda 4634-41$ emission lines and a strong He II $\lambda 4686$ absorption line, which verifies the addition of the ((f)) suffix. We compute $M_{V}=-5.45 \pm 0.22$, from $V=6.74$ and $E(B-V)=0.40$, which corresponds to a MS star, although we note that $M_{V}$ is somewhat brighter than the theoretical value $\left(M_{V}^{\text {theo }}=-5.12\right)$ of Martins \& Plez (2006) for typical $05.5 \mathrm{~V}$ stars. The difference could result from the distance taken at $1.55 \mathrm{kpc}$, or could also reflect the presence of a companion. We conclude that the spectral type of HD 46150 is $05.5 \mathrm{~V}((\mathrm{f}))$. Despite the lack of a clear periodicity or clear detection of a companion, several pieces of evidence (e.g., double peaked structures in TVS, large $\Delta R V, \ldots$ ) lead us to consider HD 46150 as a potential binary.

\subsection{Presumably single stars}

\subsubsection{HD 46056}

In the past, HD 46056 was generally considered as a spectroscopic binary because of its variable RV (Walborn 1973; Liu et al. 1989; Underhill \& Gilroy 1990). The line profiles always appear broad and shallow, suggesting the star is a single rapid rotator rather than a spectroscopic binary. The Helium lines indeed display very broad profiles extending over more than $15 \AA$ with a clearly non-Gaussian shape (see Fig. 7). Furthermore, some metallic lines, such as Mg II $\lambda 4481$ or O III $\lambda$ 5592, are too shallow to allow a measurement of their RVs by Gaussian fits or are too heavily blended to be distinguished as is the case for the C IV $\lambda \lambda$ 5801-12 lines. As a consequence, we adopted a different technique to measure the RVs. We first computed the Fourier transform of different Helium line profiles using our highest quality spectrum of the star, which combines a high signal-tonoise ratio with a high dispersion. We then found the mean projected rotational velocity $(v \sin i)$ to be $355 \pm 21 \mathrm{~km} \mathrm{~s}^{-1}$.

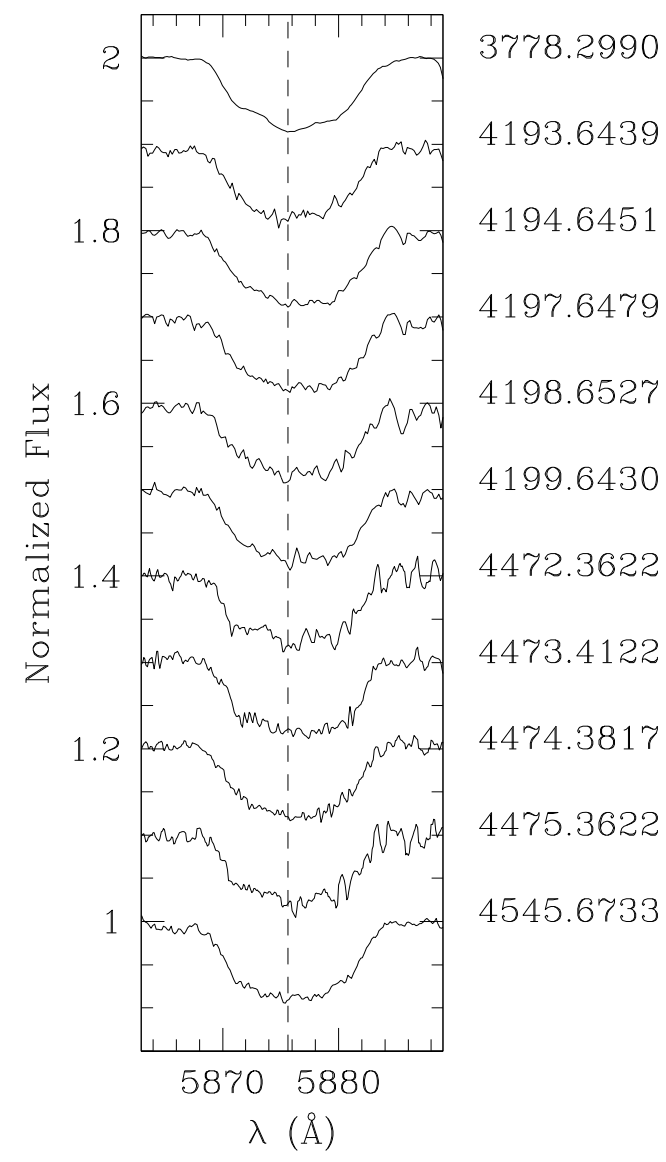

Fig. 7. He I $\lambda 5876$ line profiles of HD 46056 observed from February 2006 to March 2008.

Synthetic rotation profiles with $v \sin i=346,350,358$, and $365 \mathrm{~km} \mathrm{~s}^{-1}$ were subsequently used to fit the $\mathrm{He} \mathrm{I} \lambda 4471$, He II $\lambda$ 4542, He II $\lambda$ 4686, and He I $\lambda 5876$ lines, respectively. For all these lines, the mean of the differences between the two extreme RV values were $28.1 \mathrm{~km} \mathrm{~s}^{-1}$ and the mean $1-\sigma$ dispersion is close to $9.5 \mathrm{~km} \mathrm{~s}^{-1}$. This is close to the adopted measurement error for these broad lines. However, the line profiles appear to vary significantly as confirmed by the TVS analysis. This is reminiscent of HD 326331 (see Sana et al. 2008) and we therefore conclude that the lines of HD 46056 display some variations, although these cannot be easily associated with the presence of a companion. For instance, non-radial pulsations could be responsible for changes in the line profiles of HD 46056, which are also then likely to perturb the measurement of the radial velocities. More spectra and most of all dedicated monitoring (spectroscopic as well as photometric) with a high temporal resolution are necessary to probe the existence of these shorttime variations attributable to non-radial pulsations. The detection of these variations in the line profiles of rapid rotators was, for instance, reported by De Becker et al. (2008).

To estimate the spectral classification, since the line profiles are clearly not Gaussian, we measured the EWs by integrating the line profiles. Conti's criteria yield a spectral type O8 V. Moreover, the visual absolute magnitude of this object, calculated using $V=8.16$ and $E(B-V)=0.47$, is about $M_{V}=-4.25 \pm 0.21$, which corresponds to a MS star as suggested by the $E W \mathrm{~s}$ of the classification lines. Unlike Frost \& Conti (1976) who noticed some sporadic emission in the $\mathrm{H} \alpha$ line and assigned a spectral designation $e$ for this star, we report that the narrow emission, visible in the Balmer $\mathrm{H} \beta$ and $\mathrm{H} \alpha$ lines, 
is probably caused by the presence of nebular material around HD 46056 . This conclusion is supported by the presence of other nebular lines, e.g., the [O III] $\lambda 5007$ emission line. Therefore, a spectral type $\mathrm{O} 8 \mathrm{~V} \mathrm{n}$ is assigned to HD 46056 , in agreement with Walborn (1973) or Underhill \& Gilroy (1990).

\subsubsection{HD 46202}

Although some authors claim that HD 46202 may be RV variable (Abt \& Biggs 1972; Underhill \& Gilroy 1990), we found no variation from our two-year spectroscopic campaign (between January 2006 and January 2008) and we therefore conclude that HD 46202 is a presumably single star. Indeed, the RV dispersions are similar to those measured for the interstellar lines and the average RVs agree with those reported in the literature. Our results therefore support the previous investigations made by Plaskett \& Pearce (1931) and Petrie \& Pearce (1961). The projected rotational velocity of HD 46202, estimated using the Fourier method, amounts on average to $v \sin i=54 \pm 15 \mathrm{~km} \mathrm{~s}^{-1}$ for the stellar lines. Previous studies reported HD 46202 as an O9 V star (Morgan et al. 1955, 1965; Walborn 1971; Conti \& Leep 1974; Massey et al. 1995). Our data confirm this classification. Moreover, we obtained $M_{V}=-4.13 \pm 0.21$ from $V=8.18$ and $E(B-V)=0.44$, which indeed corresponds to a MS star. As quoted in the literature (Pérez 1991; Massey et al. 1995; Maíz-Apellániz et al. 2004), the visual magnitude seems constant in the range 8.18-8.21, with the exception of Ogura \& Ishida (1981) who derived a $V$ magnitude of 8.10.

\subsubsection{HD 46223}

HD 46223 is the hottest member of NGC 2244. The star has been regularly observed by studies of the interstellar medium (e.g., Zagury 2001), but the question of the variability was asked as early as the 1970's. Underhill \& Gilroy (1990) concluded on the basis of both published data (Cruz-González et al. 1974; Conti et al. 1977; Gies 1987) and their own observations that the star may vary. The hypothesis of a spectroscopic binary was supported by the study of Liu et al. (1989), who measured a RV dispersion of $10.3 \mathrm{~km} \mathrm{~s}^{-1}$ for three observations. However, these RV changes could also be interpreted as motions in the atmosphere of the star (Underhill \& Gilroy 1990).

The dispersions of the RVs of the more intense lines such as He II $\lambda$ 4542, He II $\lambda$ 4686, and He I $\lambda 5876$ are similar to those of the DIB. These values are slightly smaller than those of the metallic lines and the $\mathrm{He}$ I $\lambda 4471$ line. In any case, the dispersion is never significantly large even for these metallic lines: indeed, the higher amount of noise in these faint lines might explain their slight larger RV dispersion.

In consequence, we also applied the TVS method to our spectral time series. We performed this test with, on the one hand, only the Aurélie data, and, on the other hand, with all collected spectra (processed to correspond to similar spectral resolution, see Sect. 3.2.1). In both cases, we failed to detect any significant variations around the $\mathrm{He}$ I $\lambda 4471, \lambda 5876$, He II $\lambda 4542$ and near the CIV $\lambda \lambda 5801-12$ lines (Fig. 8) and we therefore considered the star to be constant.

To determine the projected rotational velocity of the star, we used the Fourier transform on the same lines as for HD 46150. We measured a mean projected rotational velocity of about $v \sin i=100 \pm 17 \mathrm{~km} \mathrm{~s}^{-1}$, while the literature reports a value of $v \sin i=103 \mathrm{~km} \mathrm{~s}^{-1}$ (Penny 1996). HD 46223 was previously classified as O5 (Hiltner 1956; Johnson 1962; Bisiacchi et al. 1982), O4 (Morgan et al. 1965), O5 ((f)) (Conti \& Leep 1974),

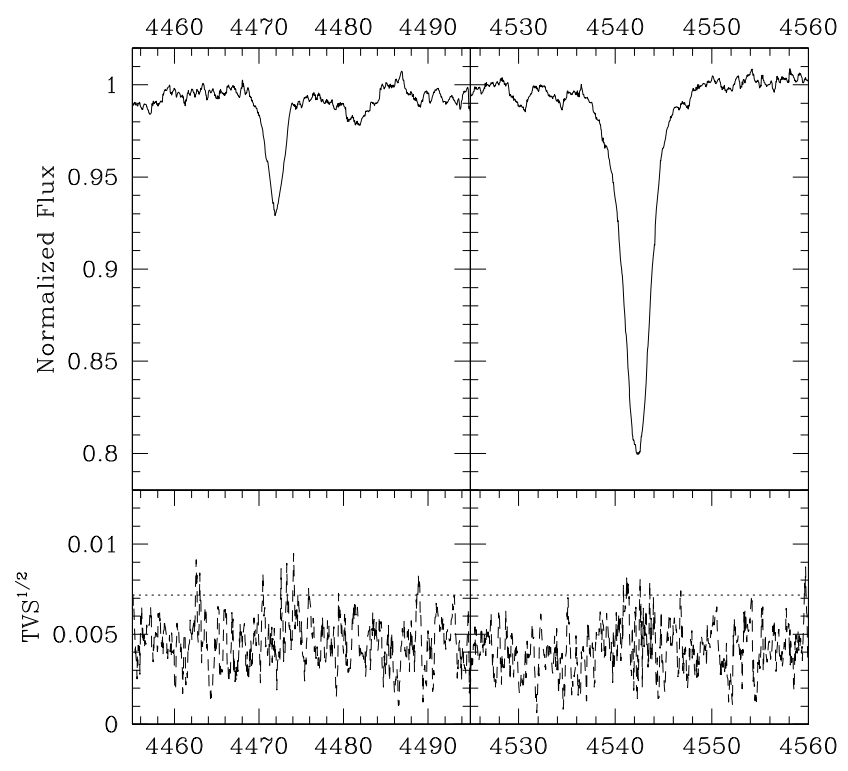

Fig. 8. Mean spectrum and TVS of HD 46223 computed from the Aurélie data for the He I $\lambda 4471$ (on the left) and the He II $\lambda 4542$ lines (on the right). The dotted line illustrates the 99\% significance level for the variability evaluated following the approach of Fullerton et al. (1996).

and, finally, as O4 V ((f)) by Massey et al. (1995). The measured $E W \mathrm{~s}$ correspond to an $\mathrm{O} 4$ star. Using $V=7.27$ and $E(B-V)=0.50$, we computed a visual absolute magnitude of about $M_{V}=-5.23 \pm 0.22$, favoring a MS classification even though this value is slightly brighter than the theoretical one $\left(M_{V}^{\text {theo }}=-5.56\right.$, Martins \& Plez 2006). The spectrum clearly exhibits strong N III $\lambda \lambda 4634-41$ emission lines accompanied by strong He II $\lambda 4686$ absorption. HD 46223 also displays both the Si IV $\lambda \lambda 4088$ and 4116 lines in emission. Therefore, we assigned an $\mathrm{O} 4 \mathrm{~V}\left(\left(\mathrm{f}^{+}\right)\right)$spectral type. We also detected weak emission in the $\mathrm{H} \alpha$ line probably due to the nebular material surrounding the star as we already mentioned for HD 46056.

\subsubsection{HD 46485}

In previous works on NGC 2244, little attention was paid to HD 46485. The literature contains only a few measurements but in no way the results of a dedicated observing run. As for HD 46056 , HD 46485 presents very broad and moderately deep lines suggesting that this star is a rapid rotator (Fig. 9).

In order to determine the projected rotational velocity of HD 46485, we independently applied a Fourier transform to three different lines, i.e., He II $\lambda$ 4686, O III $\lambda$ 5592, He I $\lambda$ 5876, and we found a mean value of $301 \pm 25 \mathrm{~km} \mathrm{~s}^{-1}$. Since the line profiles are not Gaussian, we used this measurement to generate several theoretical rotation profiles with $v \sin i=325,318$, 291 , and $301 \mathrm{~km} \mathrm{~s}^{-1}$, which we correlated with the observed He I $\lambda$ 4471, He II $\lambda$ 4542, He II $\lambda$ 4686, and He I $\lambda 5876$ lines, respectively, to derive the RVs. The $1-\sigma$ range of these lines are similar to each other (see Table 3) and, as for HD 46056 , similar to the RV measurement error. However, obvious profile changes are clearly detectable, although they are difficult to attribute to a possible binarity (Fig. 9). They may be due to non-radial pulsations.

Conti \& Leep (1974) identified HD 46485 as an O7.5 star, while Walborn (1971) suggested an O8 Vn(e) classification. Using Conti's criteria, we inferred a spectral type of $\mathrm{O} 8 \mathrm{~V}$ for 


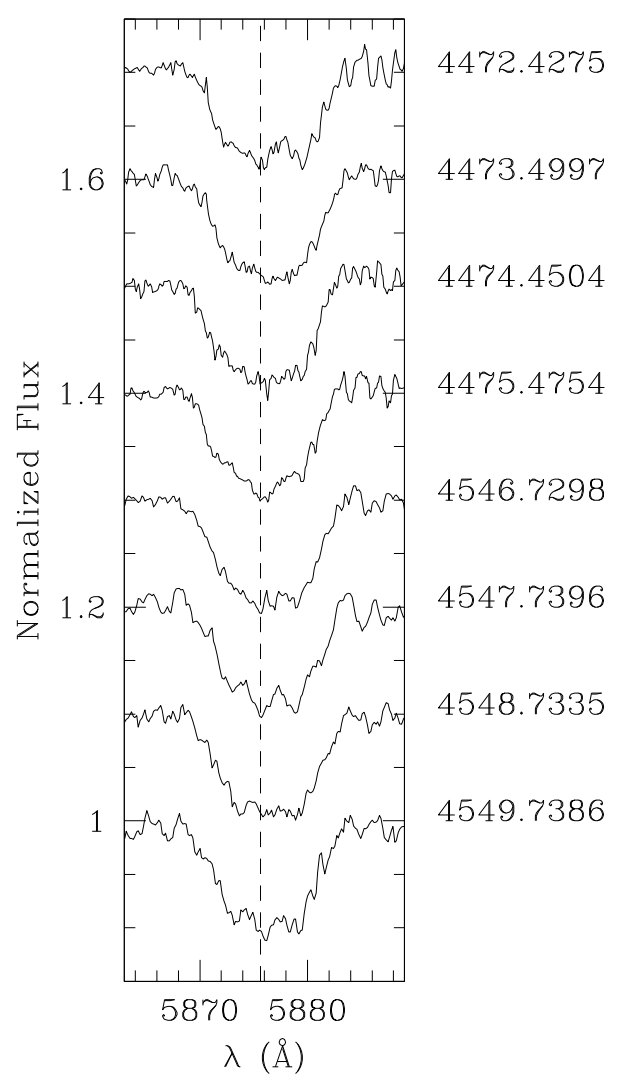

Fig. 9. Same as Fig. 7, but for HD 46485.

HD 46485 . With $V=8.20$ and $E(B-V)=0.58$, we derived a visual absolute magnitude $M_{V}=-4.68 \pm 0.36$. This value agrees with that of a typical O8 V star (Martins \& Plez 2006). In consequence, with its broad diffuse lines, we classify HD 46485 as an $\mathrm{O} 8 \mathrm{~V}$ n star.

\subsubsection{HD 46966}

For our study of the variability of HD 46966, we collected 22 spectra between January 2003 and January 2008. HD 46966 does not exhibit any significant RV dispersion in neither its metallic nor Helium lines $(\lambda 4471, \lambda 4542, \lambda 4686$, and $\lambda 5876)$. Moreover, no significant variation is observed by the TVS method. This suggests that HD 46966 is a presumably single star.

Penny (1996) estimated the projected rotational velocity of HD 46966 close to $59 \mathrm{~km} \mathrm{~s}^{-1}$. Later on, Munari \& Tomasella (1999) revised the value to be $90 \mathrm{~km} \mathrm{~s}^{-1}$. From four different lines (already mentioned for HD 46573), we measured the $v \sin i$ with the Fourier method and the derived mean value was estimated to be $63 \pm 17 \mathrm{~km} \mathrm{~s}^{-1}$ in good agreement with the value given by Penny (1996).

The spectral types quoted in the literature are O8 V (Munari \& Tomasella 1999; Maíz-Apellániz et al. 2004) or O8.5 V (Conti et al. 1977; Garmany et al. 1980). Based on the Conti criteria, we derived an $08.5 \mathrm{~V}$ spectral type. Although located outside NGC 2244, we assumed that HD 46966 is situated approximately at the same distance as the open cluster $(1.4-1.7 \mathrm{kpc})$. As before, we computed, for $V=6.87$ and $E(B-V)=0.21$, both the minimum visual absolute magnitude $M_{V}=-4.51$ and its maximum value at about $M_{V}=-4.93$. These two values are brighter than the theoretical value $\left(M_{V}^{\text {theo }}=-4.27\right.$,
Martins \& Plez 2006). However, we consider this difference to be an effect of the uncertain distance rather than a consequence of binarity, and we propose a MS classification for HD 46966.

\subsubsection{HD 48279}

Previous spectroscopic investigations by Petrie \& Pearce (1961) reported a mean radial velocity of $31.0 \pm 5.1 \mathrm{~km} \mathrm{~s}^{-1}$. Underhill \& Gilroy (1990), on the basis of their measured RV and also those of Petrie \& Pearce (1961), Conti et al. (1977) and Sanford \& Merrill (1938), proposed that the star was a single-lined spectroscopic binary.

We analyzed 4 spectra in the yellow domain and 10 in the blue region, along with 6 échelle spectra covering the entire visible domain, including the blue and yellow wavebands (see Table 1). The differences between RVs measured for Helium lines are not large enough to support the binary scenario. Although the variations are much stronger in the fainter metallic lines than in the stronger He I $\lambda 4471$ and $\lambda 5876$ lines, the accuracy in the RV measurements of the more shallow lines is more affected by the noise. In addition, no signature of a companion, such as asymmetries in line profiles, has been found in any spectrum of our time series. The TVS method, first applied to spectra in the blue domain and then to the data in the yellow region, did not show any significant variations in He I $\lambda$ 4471, He I $\lambda 5876$ nor the metallic lines. The likelihood that HD 48279 is a binary thus remains low.

We estimated the projected rotational velocity from the Fourier method to $v \sin i=130 \pm 13 \mathrm{~km} \mathrm{~s}^{-1}$. This value agrees with that of $123 \mathrm{~km} \mathrm{~s}^{-1}$ determined by Conti \& Ebbets (1977) and Penny (1996).

The spectrum of HD 48279 presents, beside the Balmer lines, deeper He I lines than He II ones, the obvious signature of a late O-type star, and we infer an O7.5 classification from Conti's criteria. We also find that Si IV $\lambda \lambda 4088-4116$ correspond to a deep absorption. The most remarkable features of the spectrum are the prominent N III absorption lines at 4195, 4634, and $4641 \AA$, implying an ON spectral designation.

Ogura \& Ishida (1981) did not mention that HD 48279 was a member of NGC 2244 but it still belongs to the Mon OB2 association (de Wit et al. 2004). Without more information, we consider the star to be at the same distance as NGC 2244. Using $V=7.91$ and $E(B-V)=0.407$, we compute the two extreme visual absolute magnitudes for a distance between 1.4 and $1.7 \mathrm{kpc}$. We obtain $M_{V}=-4.08$ and $M_{V}=-4.50$, respectively, situating HD 48279 on the MS band. Accounting for the strength of the nitrogen lines, we hence adopt the ON7.5 V spectral type for HD 48279 , slightly earlier than previous estimates (ON8 V, Conti \& Leep 1974; O8 V, Walborn 1973).

\subsection{Photometry and close "visual" companions}

In addition to our results, it is interesting to consider the photometric variability of each star. From the Hipparcos and the ASAS (All Sky Automated Survey) databases, we thus retrieved data for each studied object. These two datasets were analyzed independently since the instrumentation used in the each survey was quite different.

Table 5 lists the mean $V$ magnitude of each star and the 1- $\sigma$ standard deviation (on $V$ ) of the highest quality data provided for each star in the ASAS catalog. The Fourier transform applied to each studied object reveals no significant periodicity, concluding 
Table 5. The mean $V$ magnitude and the 1- $\sigma$ standard deviation.

\begin{tabular}{lcc}
\hline \hline Star & Mean $V$ magnitude & $1-\sigma$ error \\
\hline HD 46 056 & 8.132 & 0.006 \\
HD 46 149 & 7.598 & 0.006 \\
HD 46 150 & 6.720 & 0.021 \\
HD 46 202 & 7.946 & 0.048 \\
HD 46 223 & 7.270 & 0.006 \\
HD 46 485 & 8.255 & 0.011 \\
HD 46 573 & 7.925 & 0.008 \\
HD 46 966 & 6.854 & 0.019 \\
HD 48 279 & 7.596 & 0.026 \\
\hline
\end{tabular}

therefore that photometric variations in the stars are absent on the observed time series.

The Hipparcos catalog provides us with a sufficient number of data for only four stars of our sample: HD 46150, HD 46485 , HD 46573, and HD 46966. For these objects, we compute a mean magnitude and a corresponding 1- $\sigma$ standard deviation in the magnitude of $6.787 \pm 0.007,8.342 \pm 0.023,8.030 \pm 0.011$, and $6.851 \pm 0.007$, respectively. Once again, these small dispersions do not allow us to conclude anything about the existence of any significant variability in any of studied stars.

The speckle interferometric campaign of Galactic O-type stars with $\mathrm{V} \lesssim 8$ undertaken by Mason et al. (1998) revealed that HD 46150 and HD 48279 have "visual" companions whose separations range from $2.7^{\prime \prime}-74.6^{\prime \prime}$ and $4.6^{\prime \prime}-56.2^{\prime \prime}$, respectively. However, we note that the "visual" companion close to HD 48279 was quoted as being optical and not physical by Lindroos (1985). In addition, the Washington Visual Double Star (WDS, Mason et al. 2001) catalog also reported a "visual" companion located between 9.6" and 10.5" of HD 46056. However, we emphasize that no evidence exists so far to confirm with any certainty the physical association of these companions, especially with such high angular separations.

Turner et al. (2008) published results of an adaptive optics survey to search for faint companions around Galactic Ostar systems (with $V \lesssim 8$ ). They observed in the $I$-band to detect companions in the projected separation between $0.5^{\prime \prime}$ and 5.0". They observed five stars in our sample (HD 46149 , HD 46 150, HD 46223, HD 46 966, and HD 48279) but found no companion.

\section{Discussion}

\subsection{Multiplicity of O-type stars}

In this paper, we have studied all six O-stars of NGC 2244: HD 46 056, HD 46 149, HD 46150, HD 46 202, HD 46 223, and HD 46 485. HD 46149 is clearly a long-period SB2, while HD 46150 is a good binary candidate. The minimum frequency of spectroscopic binaries among O-stars in this cluster is thus at least $17 \%$ and could possibly reach $33 \%$.

\subsubsection{The detection biases}

Because of the spectroscopic approach, a bias exists towards the detection of $\mathrm{O}+\mathrm{OB}$ binary systems, providing only a lower limit to the true binary fraction. Several observational biases could make us miss a binary system with either a low orbital inclination, a rather large mass and/or brightness ratio, or a very wide separation (and either very long orbital period or a large eccentricity).
We first adopt the approach of Garmany et al. (1980) to estimate the probability that we have missed a binary system because of a low orbital inclination as a function of the assumed mass ratio and orbital period. In this scheme, we consider that the semi-amplitude of the $R V$ curve $K$ should be smaller or equal to twice the $R V$ dispersion $\left(\sigma_{R V}\right)$ of the photospheric lines, i.e., He II $\lambda 4542$ in the blue domain and C IV $\lambda 5812$ in the yellow region (or He I $\lambda 5876$ when this metallic line is not visible). The mass function of the primary component is given by

$$
\begin{aligned}
f(m) & =\frac{M_{1} \sin ^{3} i}{q(1+q)^{2}} \\
& =1.0355 \times 10^{-7} K^{3} P\left(1-e^{2}\right)^{3 / 2}
\end{aligned}
$$

where $M_{1}$ is the primary mass (in $M_{\odot}$ ), $K$ is expressed in $\mathrm{km} \mathrm{s}^{-1}$, $e$ represents the orbital eccentricity, $q=M_{1} / M_{2}$, and $P$ defines the orbital period (in days).

Equation (2) is transformed to express the orbital inclination as a function of other parameters. To obtain an upper limit to $\sin i$, we insert $2 \sigma_{R V}$ as an upper limit to $K$ and assume a zero eccentricity. Therefore, we obtain

$\sin i \leq 9.392 \times 10^{-3} \sigma_{R V}\left(\frac{P q(1+q)^{2}}{M_{1}}\right)^{1 / 3}$.

By assuming a random distribution of orbital directions in space, we can write the probability that the orbital inclination is lower than the value obtained from Eq. (3) as

$$
\int_{0}^{i_{\text {up }}} \sin i \mathrm{~d} i=1-\cos i_{\text {up }}
$$

The probabilities reported in Table 6 are the mean values computed from the two photospheric lines quoted above and by using as primary masses, the masses listed in Martins et al. (2005).

The probabilities that HD 46202, HD 46 966, and HD 48279 $\left(\simeq 20 M_{\odot}\right)$ are binaries with orbital periods of less than 28 days and a companion earlier than $\mathrm{A} 5\left(\simeq 2 M_{\odot}, q=10\right)$ are very low (i.e., $\leq 7 \%$ ). Any missed binary would most probably have to be a system with a rather large mass ratio and a long orbital period.

The same conclusion could be reached for HD 46223 $\left(\simeq 47 M_{\odot}\right)$. At a period of 28 days, the probability of having an O-type companion is close to zero $(\sim 0.2 \%)$, and we have only a $3 \%$ chance of missing a B3 secondary $\left(\simeq 8 M_{\odot}, q=5\right)$. However, for the same orbital period, there is a $10 \%$ chance that the binary signature could have been missed if the spectral type of the second component was B6 or later $\left(\$ 5 M_{\odot}, q=10\right)$.

We also perfomed Monte-Carlo simulations following the method of Sana et al. (2009, submitted). The orbital parameters were randomly generated (10000 trials) based on the assumption that $50 \%$ of the binaries have an orbital period of $P \leq 10$ days, while the other $50 \%$ have $P>10$ days. Moreover, the periods are uniformly drawn from $\log P$ space (between 0.3 and 3.5), the system orientations $(\cos i)$ are randomly drawn from a uniform distribution, the mass ratio $q=M_{1} / M_{2}$ is also uniformly distributed between 0.1 and 1.0 , and we uniformly selected eccentricities of between 0.0 and 0.8 . The simulations show that the probability (Table 7) of have missing, in HD 46202 and HD 46223, a peak-to-peak RV variation $(\Delta \mathrm{RV})$ larger than the observed value of $20 \mathrm{~km} \mathrm{~s}^{-1}$ is less than $1 \%$ for systems with $2 \leq P \leq 10$ days and less than $10 \%$ for $10 \leq P \leq 365$ days. For the rapid rotators, the simulations were run for $\Delta R V>$ $40 \mathrm{~km} \mathrm{~s}^{-1}$ since the measurement errors are larger. The probabilities amount to $2 \%$ and $20 \%$ for HD 46056 and HD 46 485, respectively. Therefore, it is very unlikely that short-period $\mathrm{O}+\mathrm{OB}$ 
Table 6. Probability to have missed a binary system ${ }^{a}$.

\begin{tabular}{|c|c|c|c|c|c|c|c|c|c|c|c|c|c|c|c|}
\hline & \multicolumn{5}{|c|}{$P=5$ days } & \multicolumn{6}{|c|}{$P=14$ days } & \multicolumn{4}{|c|}{$P=28$ days } \\
\hline & $q=1$ & \multicolumn{2}{|c|}{$q=5$} & \multicolumn{2}{|c|}{$q=10$} & \multicolumn{2}{|c|}{$q=1$} & \multicolumn{2}{|c|}{$q=5$} & \multicolumn{2}{|c|}{$q=10$} & \multicolumn{2}{|c|}{$q=1$} & $q=5$ & $q=10$ \\
\hline HD 46056 & $3.9 \mathrm{E}-3$ & \multicolumn{2}{|c|}{$5.0 \mathrm{E}-2$} & \multicolumn{2}{|c|}{$19.4 \mathrm{E}-2$} & \multicolumn{2}{|c|}{$7.8 \mathrm{E}-3$} & \multicolumn{2}{|c|}{$10.3 \mathrm{E}-2$} & \multicolumn{2}{|c|}{$44.9 \mathrm{E}-2$} & \multicolumn{2}{|c|}{$1.2 \mathrm{E}-2$} & $16.9 \mathrm{E}-2$ & \\
\hline HD 46150 & $3.6 \mathrm{E}-3$ & \multicolumn{2}{|c|}{ 4.6E-2 } & \multicolumn{2}{|c|}{$18.1 \mathrm{E}-2$} & \multicolumn{2}{|c|}{ 7.1E-3 } & \multicolumn{2}{|c|}{$9.5 \mathrm{E}-2$} & \multicolumn{2}{|c|}{$45.5 \mathrm{E}-2$} & \multicolumn{2}{|c|}{$1.1 \mathrm{E}-2$} & $15.7 \mathrm{E}-2$ & $34.7 \mathrm{E}-2$ \\
\hline HD 46202 & $4.7 \mathrm{E}-4$ & \multicolumn{2}{|c|}{$6.0 \mathrm{E}-3$} & \multicolumn{2}{|c|}{$2.2 \mathrm{E}-2$} & \multicolumn{2}{|c|}{$9.4 \mathrm{E}-4$} & \multicolumn{2}{|c|}{$1.2 \mathrm{E}-2$} & \multicolumn{2}{|c|}{ 4.3E-2 } & \multicolumn{2}{|c|}{$1.5 \mathrm{E}-3$} & $1.9 \mathrm{E}-2$ & 7.0E-2 \\
\hline HD 46223 & $6.4 \mathrm{E}-4$ & \multicolumn{2}{|c|}{ 8.1E-3 } & \multicolumn{2}{|c|}{$2.9 \mathrm{E}-2$} & $1.3 \mathrm{E}$ & & $1.6 \mathrm{I}$ & & & & $2.0 \mathrm{I}$ & & $2.6 \mathrm{E}-2$ & $9.7 \mathrm{E}-2$ \\
\hline HD 46485 & $5.1 \mathrm{E}-3$ & $6.7 \mathrm{I}$ & & 26. & E-2 & $1.0 \mathrm{E}$ & & 13.8 & & & -1 & $1.6 \mathrm{I}$ & & $23.1 \mathrm{E}-2$ & \\
\hline HD 46966 & $3.4 \mathrm{E}-4$ & $4.3 \mathrm{I}$ & & & $\mathrm{E}-2$ & $6.8 \mathrm{E}$ & & $8.6 \mathrm{I}$ & & & & $1.1 \mathrm{I}$ & & $1.4 \mathrm{E}-2$ & $5.0 \mathrm{E}-2$ \\
\hline HD 48279 & $2.3 \mathrm{E}-4$ & $2.9 \mathrm{I}$ & & & -2 & $4.5 \mathrm{E}$ & & $5.7 \mathrm{I}$ & & & & $7.1 \mathrm{I}$ & & $9.0 \mathrm{E}-3$ & $3.3 \mathrm{E}-2$ \\
\hline & & & & & $\overline{P=t}$ & $\overline{\overline{\text { days }}}$ & & & & & $=3$ & o day & & & \\
\hline & & & & & & & & & & & & & & & \\
\hline & HD 46 & & & & 30. & & & & & & & & & & \\
\hline & HD 46 & & & & 28. & & 78.2 & E-2 & & & 53.3 & & & & \\
\hline & HD 46 & & & & & & 12. & E-2 & & & & & & E-2 & \\
\hline & HD 46 & & & & & & 17.2 & E-2 & & & 13.7 & & & E-2 & \\
\hline & HD 46 & & & & 43. & & & & & & & & & & \\
\hline & HD 46 & 66 & & & & & & E-2 & & & & & & E-2 & \\
\hline & HD 48 & & 1.2 & & 1.5 & & 5.5 & E-2 & & -3 & 4.5 & & & E-2 & \\
\hline
\end{tabular}

${ }^{a}$ Table 6 reports the probabilities that a binary system could have been missed for different values of the orbital period $P$ and of the mass ratio $q=M_{1} / M_{2}$

systems were missed by our study, and this result does not depend on the small number of stars in NGC 2244. For long-period (365-3000 days) systems, the probability of missing binaries increases to $50 \%$ ( $70 \%$ for rapid rotators). This is expected since our sampling does not permit us to investigate thoroughly this part of the parameter space.

\subsubsection{The effects of stellar evolution}

We now compare our results with those of other young open clusters studied in a similar way. The minimal binary fraction in NGC $2244(17 \%)$ appears close to that of IC $1805(20 \%)$. However, we note differences in the stellar content of these two clusters. De Becker et al. (2006) found that at least one giant (O III (f)) and one supergiant (O If) belong to IC 1805, while all O-type stars in NGC 2244 are MS objects. Evolution effects (and thus the age of the systems) therefore seem to play no role in the binary properties, although this conclusion should be confirmed by studies of other clusters.

García \& Mermilliod (2001) asserted that the orbital periods of spectroscopic binaries in the O-type star rich clusters were around 4-5 days. We emphasize that the absence of short-period binaries in NGC 2244 does not support this conclusion (see previous subsection).

A comparison between NGC 2244, IC 1805, and NGC 6231 (all three now being well-studied) is provided in Table 8. Differences are readily seen in this table. Apart from the already quoted difference in the proportion of short versus long-period binaries, there is also a difference in the nature of the companion. If we consider all O-type stars to be binaries, i.e., those apparently single stars with undetectable low-mass companions, the majority of O-stars in NGC 6231 appears to have OB companions, while the signature of additional early-type objects is found in only a few O-stars of NGC 2244 and IC 1805.

\subsubsection{A possible impact of the stellar density}

The surface density of a given cluster is evaluated to be the ratio of the number of members of the cluster to the circular area delineated by a typical radius, e.g., the one given by
Table 7. Probability of missing a significant orbital $R V$ variation $^{a}$.

\begin{tabular}{lccccc}
\hline \hline Star & $M_{1}$ & $2-10$ & $10-365$ & $365-3000$ & $2-3000$ \\
\hline HD 46 056 & 20.8 & 0.02 & 0.17 & 0.77 & 0.24 \\
HD 46 149 & 20.8 & 0.01 & 0.04 & 0.31 & 0.07 \\
HD 46 150 & 34.4 & 0.01 & 0.03 & 0.21 & 0.05 \\
HD 46 202 & 17.1 & 0.01 & 0.09 & 0.50 & 0.13 \\
HD 46 223 & 46.9 & 0.01 & 0.03 & 0.22 & 0.05 \\
HD 46 485 & 20.8 & 0.01 & 0.20 & 0.68 & 0.20 \\
HD 46 573 & 22.9 & 0.01 & 0.06 & 0.38 & 0.09 \\
HD 46 966 & 18.8 & 0.01 & 0.06 & 0.34 & 0.08 \\
HD 48 279 & 22.9 & 0.01 & 0.07 & 0.40 & 0.10
\end{tabular}

${ }^{a}$ Table 7 lists the probability of missing a significant orbital $R V$ variation $\left(\Delta R V>40 \mathrm{~km} \mathrm{~s}^{-1}\right.$ for the two rapid rotators and $\Delta R V>20 \mathrm{~km} \mathrm{~s}^{-1}$ for the other stars). $M_{1}$ represents the primary mass and is expressed in $M_{\odot}$. The last four columns are classified according to the orbital period of the system (in days).

Tadross et al. (2002). Both IC 1805 and NGC 2244 have a rather loose cluster core, whilst the stellar surface density appears much higher in the central part of NGC 6231. With the new binary fraction from De Becker et al. (2006), Sana et al. (2008), and this work, the anticorrelation between the cluster density and its massive star binary fraction proposed by García \& Mermilliod (2001) can no longer be supported. In contrast, a possible correlation might reflect the behavior detected in these three clusters more accurately. However, we must remain careful since this assumption is directly linked to the error bars in the binary fraction. If it is confirmed, this would emphasize the important role of dynamical gravitational interactions in the formation of massive binaries (Zinnecker 2003). Confirmation, however, of this relation will require more data. The extreme case of loose $\mathrm{OB}$ associations might provide a good testbed for this confirmation.

\subsection{Rotational velocities}

HD 46056 and HD 46485 are two rapid rotators whose lines appear slightly variable. Rapid rotators could either be

- born as such and maintain a rapid rotation over their mainsequence lifetime; 
Table 8. Summary of the multiplicity in young open clusters ${ }^{a}$.

\begin{tabular}{cccccccc}
\hline \hline Cluster & $\begin{array}{c}\text { Density } \\
\left(\text { stars/pc }^{2}\right)\end{array}$ & $\begin{array}{c}\text { Minimal binary } \\
\text { fraction }\end{array}$ & n\# O-stars & $\begin{array}{c}\text { Short-period } \\
\text { binaries }(P \leq 10)\end{array}$ & $\begin{array}{c}\text { Long-period } \\
\text { binaries }\end{array}$ & $\begin{array}{c}\text { Variable } \\
\text { stars }\end{array}$ & References \\
\hline NGC 2244 & 2.84 & $17 \%$ & 6 & 0 & 1 & 3 & $\begin{array}{l}\text { This paper } \\
\text { Rauw \& De Becker (2004) } \\
\text { IC 1805 }\end{array}$ \\
\hline 1.65 & $20 \%$ & 10 & 1 & 1 & $4^{\star}$ & $\begin{array}{l}\text { De Becker et al. (2006) } \\
\text { Sana et al. (2008) }\end{array}$ \\
NGC 6231 & 12.46 & $63 \%$ & 16 & $6^{\star \star}$ & 4 & 3 & 3 \\
\hline
\end{tabular}

${ }^{a}$ The first and second columns give the name and the density of the cluster. The third column provides the minimal binary fraction determined after intensive monitoring of the O-type stars. The next columns present the number of O-stars in each cluster, and the number of detected short and long-period binaries and the variable stars (potential binaries and probable intrinsic variables). The last column quotes relevant references. * Notes: four late O-type stars were not studied by the two papers mentioned in the references. We will consider these objects as variable in the absence of a thorough study. ${ }^{\star \star}$ Notes: we take into account that NGC 6231 also contains a WR+O binary since a WR is an evolved O-star.

- spun-up by transfer of mass and angular momentum during a Roche lobe overflow in a binary system;

- spun-up because of the core contraction at the end of the main-sequence.

For these two rapid rotators, it seems more likely that the first hypothesis is the correct one since we found no clear indication of multiplicity and there is no evidence that these objects are at or near the end of their main-sequence lifetime (for instance, on the basis of their chemical enrichment).

We also compared our distribution of the projected rotational velocities of O-type stars in and around NGC 2244 to the corresponding distribution for B-type stars. Huang \& Gies (2006) performed a survey of projected rotational velocities for a large sample of mainly B-type (later than O9.5) main-sequence or giant stars in young open clusters, including NGC 2244. The $v \sin i$ values were derived by comparison between theoretical line profiles and observed profiles of the He I $\lambda \lambda 4026,4387$, 4471 , and $\mathrm{Mg}$ II $\lambda 4481$ lines. Huang \& Gies (2006) reported the projected rotational velocities of 41 stars in NGC 2244. The average projected rotational velocity of the B-type stars equals to $168 \mathrm{~km} \mathrm{~s}^{-1}$. The 15 stars that are earlier than B3 show an average $v \sin i$ of $153 \mathrm{~km} \mathrm{~s}^{-1}$. Five stars out of the total sample (i.e., $12 \%$ ) were found to display a projected rotational velocity of above $300 \mathrm{~km} \mathrm{~s}^{-1}$. At first sight, the distribution of the rotational velocities of the O-type stars looks different from that of the B-type stars (see Fig. 10). We performed a two-sample Kolmogorov-Smirnov test to check whether or not the two distributions are significantly different. On the basis of this test and adopting a usual significance level of 0.05 , the null hypothesis (both samples are drawn from the same parent distribution) cannot be rejected. This is true for both the full sample and the six O-stars of NGC 2244.

\section{Summary and conclusions}

Our long-term monitoring has shed new light on the multiplicity of six massive O-stars in NGC 2244 plus three in the association Mon OB2. Table 9 summarizes the present knowledge for these stars.

In the association Mon OB2, the current study reveals the detection of a new SB1: HD 46573. Although we determine a period of 10.67 days and compute the orbital parameters of the system, these results must still be considered as preliminary. Indeed, the accuracy of the RV measurements could strongly influence the orbital solution of the system, considering the low amplitude of the radial velocity variations. The eccentricity of the orbit remains poorly constrained but we note that the eccentric radial velocity curve appears slightly more likely solution than the circular one.
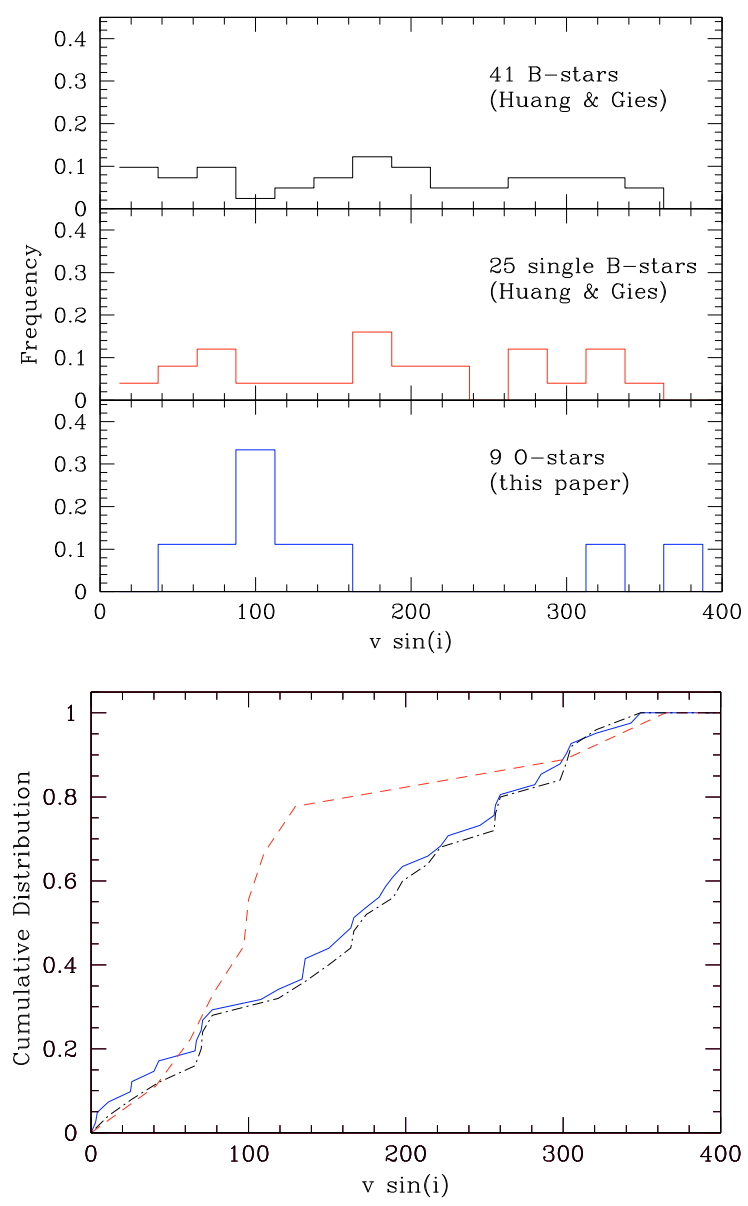

Fig. 10. Top: the distribution of projected rotational velocities of (1) the full sample of B stars studied by Huang \& Gies (2006, top panel), (2) those B stars of Huang \& Gies that show a constant radial velocity (middle panel), and (3) our sample of O-type stars. Bottom: the cumulative distributions of the three samples: all B-stars (solid line), single B stars (dashed-dotted line), and O-stars (dashed line).

Another interesting result, in NGC 2244, is the detection, for the first time, of a secondary component in HD 46 149. However, for the time being, we can only constrain the period to be at least several tens of days. By virtue of its mass and flux ratios, HD 46149 is considered to be an $\mathrm{O}+\mathrm{B}$ binary. An observation campaign should be initiated to monitor the star and constrain the orbital solution of its system more accurately. Finally, we also detect weak variations in the RVs for one star belonging to NGC 2244 (HD 46150) and variations in the line profiles, most probably unrelated to binarity, in the two rapid 
Table 9. Summary ${ }^{a}$ of the optical properties of O-stars in NGC 2244 (top part) and Mon OB2 (bottom).

\begin{tabular}{lccc}
\hline \hline Name & Spectral type & $v \sin i$ & Spectroscopic status \\
\hline HD 46 056 & O8Vn & $355 \pm 21$ & C ? (rapid rotator) \\
HD 46149 & O8V+B0-1V & $78 \pm 11^{\ddagger}$ & SB2 \\
HD 46 150 & O5.5V $((\mathrm{f}))$ & $97 \pm 9$ & Bin? \\
HD 46202 & O9V & $54 \pm 15$ & $\mathrm{C}$ \\
HD 46223 & O4V $\left(\left(\mathrm{f}^{+}\right)\right)$ & $100 \pm 17$ & $\mathrm{C}$ \\
HD 46 485 & O8Vn & $301 \pm 25$ & $\mathrm{C}$ ? (rapid rotator) \\
\hline HD 46573 & O7.5V((f)) & $110 \pm 18$ & SB1 \\
HD 46 966 & O8.5V & $63 \pm 17$ & $\mathrm{C}$ \\
HD 48 279 & ON7.5V & $130 \pm 13$ & $\mathrm{C}$ \\
\hline
\end{tabular}

${ }^{a}$ The first column provides the name of each star studied in this paper. The next two columns give the spectral classification and the projected rotational velocity (in $\mathrm{km} \mathrm{s}^{-1}$ ) of the O-type stars, while the last column reports the status of the star as derived from our campaign: " $\mathrm{C}$ " means that our study did not reveal any indication of RV variability or of binarity, suggesting the star is probably single, "Bin?" indicates a potential binary, "SB1" corresponds to a spectroscopic binary where the second component has not been detected yet but where a period has been determined, whilst "SB2" corresponds to a spectroscopic binary where the two components are visible. ${ }^{\star}$ Notes: the reported value for HD 46149 is the projected rotational velocity for the primary.

rotators (HD 46056 and HD 46 485). The observed binary fraction is thus $17 \%$ at minimum.

This work, combined to the results of studies of NGC 6231 and IC 1805, also revealed significant differences between clusters regarding the preference of $\mathrm{O}+\mathrm{OB}$ systems and the distribution of short versus long-period binaries. These differences cannot be explained by the cluster age but could be linked to density effects. Of course, to confirm such a general conclusion, it is important to rely upon a large sample of well-studied very young open clusters that harbor a significant population of early-type stars. To date however, only a few clusters have been the subject of such an intensive search for binary systems. It is therefore of paramount importance to perform similar studies on a larger sample of clusters, spanning as wide a range of physical properties as possible.

Acknowledgements. This work was supported by the FNRS (Belgium) and by a PRODEX XMM/Integral contract (Belspo) and by the Communauté française de Belgique - Action de recherche concertée (ARC) - Académie Wallonie-Europe. The travels to OHP were supported by the Ministère de l'Enseignement Supérieur et de la Recherche de la Communauté Française. Philippe Eenens acknowledges support through CONACyT grant 67041. We also thank the staff of San Pedro Màrtir Observatory (Mexico) and of Observatoire de Haute-Provence (France) for their technical support. This work made use of the SIMBAD and the ASAS and Hipparcos databases.

\section{References}

Abt, H. A., \& Biggs, E. S. 1972, Bibliography of stellar radial velocities, ed. H. A. Abt, \& E. S. Biggs

Bisiacchi, G. F., Lopez, J. A., \& Firmani, C. 1982, A\&A, 107, 252

Chen, L., de Grijs, R., \& Zhao, J. L. 2007, AJ, 134, 1368

Conti, P. S. 1973, ApJ, 179, 161

Conti, P. S., \& Alschuler, W. R. 1971, ApJ, 170, 325

Conti, P. S., \& Leep, E. M. 1974, ApJ, 193, 113

Conti, P. S., \& Ebbets, D. 1977, ApJ, 213, 438

Conti, P. S., Leep, E. M., \& Lorre, J. J. 1977, ApJ, 214, 759

Cruz-González, C., Recillas-Cruz, E., Costero, R., Peimbert, M., \& TorresPeimbert, S. 1974, RMA\&A, 1, 211
De Becker, M., Rauw, G., Manfroid, J., \& Eenens, P. 2006, A\&A, 456, 1121 De Becker, M., Linder, N., \& Rauw, G. 2008, Information Bulletin on Variable Stars, 5841, 1

de Wit, W. J., Testi, L., Palla, F., Vanzi, L., \& Zinnecker, H. 2004, A\&A, 425, 937

Feldmeier, A., Puls, J., \& Pauldrach, A. W. A. 1997, A\&A, 322, 878

Frost, S. A., \& Conti, P. S. 1976, in Be and Shell Stars, ed. A. Slettebak, IAU Symp., 70, 139

Fullerton, A. W. 1990, Ph.D. Thesis, AA(Toronto Univ. (Ontario).)

Fullerton, A. W., Gies, D. R., \& Bolton, C. T. 1996, ApJS, 103, 475

García, B., \& Mermilliod, J. C. 2001, A\&A, 368, 122

Garmany, C. D., Conti, P. S., \& Massey, P. 1980, ApJ, 242, 1063

Gies, D. R. 1987, ApJS, 64, 545

González, J. F., \& Levato, H. 2006, A\&A, 448, 283

Gosset, E., Royer, P., Rauw, G., Manfroid, J., \& Vreux, J.-M. 2001, MNRAS, 327,435

Heck, A., Manfroid, J., \& Mersch, G. 1985, A\&AS, 59, 63

Hensberge, H., Pavlovski, K., \& Verschueren, W. 2000, A\&A, 358, 553

Hiltner, W. A. 1956, ApJS, 2, 389

Huang, W., \& Gies, D. R. 2006, ApJ, 648, 591

Johnson, H. L. 1962, ApJ, 136, 1135

Kharchenko, N. V., Piskunov, A. E., Röser, S., Schilbach, E., \& Scholz, R.-D. 2005, A\&A, 440, 403

Li, J. Z., \& Smith, M. D. 2005, A\&A, 431, 925

Linder, N., Rauw, G., Martins, F., et al. 2008, A\&A, 489, 713

Lindroos, K. P. 1985, A\&AS, 60, 183

Liu, T., Janes, K. A., \& Bania, T. M. 1989, AJ, 98, 626

Maíz-Apellániz, J., Walborn, N. R., Galué, H. Á., \& Wei, L. H. 2004, ApJS, 151, 103

Martins, F., \& Plez, B. 2006, A\&A, 457, 637

Martins, F., Schaerer, D., \& Hillier, D. J. 2005, A\&A, 436, 1049

Mason, B. D., Gies, D. R., Hartkopf, W. I., et al. 1998, AJ, 115, 821

Mason, B. D., Wycoff, G. L., Hartkopf, W. I., Douglass, G. G., \& Worley, C. E. 2001, AJ, 122, 3466

Massey, P., Johnson, K. E., \& Degioia-Eastwood, K. 1995, ApJ, 454, 151

Mathys, G. 1988, A\&AS, 76, 427

Mathys, G. 1989, A\&AS, 81, 237

Morgan, W. W., Code, A. D., \& Whitford, A. E. 1955, ApJS, 2, 41

Morgan, W. W., Hiltner, W. A., Neff, J. S., Garrison, R., \& Osterbrock, D. E. 1965, ApJ, 142, 974

Munari, U., \& Tomasella, L. 1999, A\&AS, 137, 521

Ogura, K., \& Ishida, K. 1981, PASJ, 33, 149

Penny, L. R. 1996, ApJ, 463, 737

Pérez, M. R. 1991, Revista Mexicana de Astronomia y Astrofisica, 22, 99

Petrie, R. M., \& Pearce, J. A. 1961, Publications of the Dominion Astrophysical Observatory Victoria, 12, 1

Plaskett, J. S., \& Pearce, J. A. 1931, Publications of the Dominion Astrophysical Observatory Victoria, 5, 1

Rauw, G., \& De Becker, M. 2004, A\&A, 421, 693

Reed, C. 2005, VizieR Online Data Catalog, 5125, 0

Sana, H., Gosset, E., \& Rauw, G. 2006a, MNRAS, 371, 67

Sana, H., Rauw, G., Nazé, Y., Gosset, E., \& Vreux, J.-M. 2006b, MNRAS, 372, 661

Sana, H., Gosset, E., Nazé, Y., Rauw, G., \& Linder, N. 2008, MNRAS, 386, 447 Sanford, R. F., \& Merrill, P. W. 1938, ApJ, 87, 517

Simón-Díaz, S., \& Herrero, A. 2007, A\&A, 468, 1063

Stickland, D. J. 1996, The Observatory, 116, 294

Tadross, A. L., Werner, P., Osman, A., \& Marie, M. 2002, New Astronomy, 7, 553

Turner, D. G. 1976, ApJ, 210, 65

Turner, N. H., ten Brummelaar, T. A., Roberts, L. C., et al. 2008, AJ, 136, 554

Underhill, A. B., \& Gilroy, K. K. 1990, ApJ, 364, 626

Walborn, N. R. 1971, ApJS, 23, 257

Walborn, N. R. 1973, AJ, 78, 1067

Walborn, N. R., \& Fitzpatrick, E. L. 1990, PASP, 102, 379, erratum: 102, 1094

Wang, J., Townsley, L. K., Feigelson, E. D., et al. 2008, ApJ, 675, 464

Wolfe, Jr., R. H., Horak, H. G., \& Storer, N. W. 1967, The machine computation of spectroscopic binary elements, ed. M. Hack, 251

Zagury, F. 2001, New Astron., 6, 403

Zinnecker, H. 2003, in A Massive Star Odyssey: From Main Sequence to Supernova, ed. K. van der Hucht, A. Herrero, \& C. Esteban, IAU Symp., 212, 80

Zinnecker, H., \& Yorke, H. W. 2007, ARA\&A, 45, 481 